\title{
A coupled ecological-hydrodynamic model for the spatial distribution of sessile aquatic species in thermally forced basins
}

\author{
Lorenzo Mari $^{\mathrm{a}, *}$, Cristian Biotto ${ }^{\mathrm{b}, 1}$, Astrid Decoene $^{\mathrm{b}, 2}$, Luca Bonaventura $^{\mathrm{b}}$ \\ a Dipartimento di Elettronica e Informazione, Politecnico di Milano, Via Ponzio 34/5, 20133 Milano, Italy \\ b MOX, Dipartimento di Matematica “F. Brioschi”, Politecnico di Milano, Via Bonardi 9, 20133 Milano, Italy
}

\section{A R T I C L E I N F O}

\section{Article history:}

Received 23 December 2008

Received in revised form 6 May 2009

Accepted 9 May 2009

Available online 15 June 2009

\section{Keywords:}

Larval dispersal

Diffusion patterns

Connectivity matrix

Hydrodynamics

Thermally forced flows

Limnology

Lagrangian modeling

Numerical methods

\begin{abstract}
A B S T R A C T
The life cycle of several sessile or highly sedentary aquatic species is characterized by a pelagic stage, during which propagules are dispersed by the water flow. As a consequence, hydrodynamics plays a crucial role in redistributing offspring. In this work, we describe an integrated modeling framework that couples a minimal - yet biologically well founded - ecological model for the population dynamics at the local scale to an efficient numerical model of three dimensional free surface flows in a thermally forced basin. The computed hydrodynamical fields are employed in a Lagrangian description of larval transport at the basin scale. The developed modeling framework has been applied to a realistic case study, namely the spread of an idealized aquatic sedentary population in Lake Garda, Italy. The analysis of this case study shows that the long-term interplay between demography and hydrodynamics can produce complex spatiotemporal dynamics. Our results also evidence that larvae can travel over relatively long distances even in a closed basin. A sensitivity analysis of the model outcomes shows that both biological traits and external forcings may remarkably influence the evolution of diffusion patterns in space and time.
\end{abstract}

(C) 2009 Elsevier B.V. All rights reserved.

\section{Introduction}

One of the most important and challenging problems in population ecology is the understanding of the spatial dynamics of animal populations. This represents indeed a very complex task, since it requires the study of processes occurring at different spatial scales, ranging from local ones, such as birth and death processes, to non-local ones, such as dispersal processes (Levin, 1992). A case of particular interest is represented by those aquatic species that are sessile or highly sedentary as adults, but have a pelagic stage at the beginning of their life cycle (e.g., mussels, corals, etc.; see Guichard et al., 2004; Cowen and Sponaugle, 2009). For these species, the redistribution of organisms between subsequent generations is almost exclusively operated by the water flow. The resulting patterns of larval dispersal can thus be extremely complex, since they reflect the effects of the underlying hydrodynamics (Becker et al., 2007; Cowen and Sponaugle, 2009; Siegel et al., 2008), and play

\footnotetext{
* Corresponding author. Tel.: +39 022399 3406; fax: +39 0223993412. E-mail addresses: lorenzo.mari@polimi.it (L. Mari), c.biotto07@imperial.ac.uk (C. Biotto), astrid.decoene@gmail.com (A. Decoene), luca.bonaventura@polimi.it (L. Bonaventura).

1 Present address: Imperial College, London, UK.

2 Present address: Université Paris Sud, Paris, France.
}

a key role in determining long-term spatiotemporal population dynamics.

Both numerical simulations and measurements of dispersal abilities have contributed to better understanding of some features of larval transport in marine and freshwater ecosystems (Sammarco and Andrews, 1988; Cowen et al., 2000). The outcomes of numerical models have been sometimes profitably compared to those of genetic models (Galindo et al., 2006), elemental fingerprinting techniques (Shank and Halanych, 2007) or in situ larval culturing (Becker et al., 2007). Nevertheless, coupled ecological-hydrodynamic models are still needed in order to understand and forecast spatial population dynamics in a comprehensive way, as first clearly stated by Roughgarden et al. (1988). Such models may in fact contribute to answering the basic questions concerning larval dispersal, i.e., where do larvae go and where do larvae come from (Levin, 2006). Although questions of this kind could seem even too naive, they correspond de facto to some of the most debated topics in the literature on larval dispersal, such as the estimation of the spatial scale over which larval transport occurs (Cowen et al., 2006) and the relevance of self-recruitment (that is, the retention of larvae in their native site) on population dynamics at the basin scale (Cowen et al., 2000, 2006; Becker et al., 2007). A comprehensive understanding of spatiotemporal recruitment patterns is also required to face important applied issues, such as population management and the design of protected areas (Guichard et al., 2004; Werner et al., 2007; Cowen and Sponaugle, 2009). 
In this work, we propose an integrated modeling framework that combines a minimal (yet ecologically well-founded) population model for the local demographic dynamics of a spatially structured sessile population with a rigorous description of transport effects. In particular, we aim to describe long-term population dynamics. This actually represents a necessary condition to understand the interplay between hydrodynamic and demographic processes and the resulting spatiotemporal patterns of population abundance at the basin scale. Remarkably, very few works have reported the results of long-term analyses in the context of larval dispersal. In point of fact, a recent literature survey (Miller, 2007) has reported that just 5 on the 69 reviewed papers on fish recruitment in marine ecosystems were multigenerational (e.g., Rose et al., 2003; see also Bode et al., 2006). Moreover, most works devoted to the analysis of larval dispersal refer to marine ecosystems, while we specifically address the study of closed water bodies. Larval dispersal is described here by a Lagrangian approach. The velocity fields used to evaluate larval trajectories are computed using the volume-conservative finite element model described in Miglio et al. (1999) and Causin and Miglio (2002), subsequently extended in Biotto (2007) to include baroclinic effects due to thermal forcing induced by solar radiation. The resulting coupled model is applied to a case study concerning the spread of an ideal sessile organism in Lake Garda, Italy. Our analysis demonstrates that demography and hydrodynamics work together to produce complex spatiotemporal dynamics. We also show that the presented results are quite robust to changes in the modeling assumptions, while biological parameters and environmental forcing terms may sensibly affect the long-term evolution of spatiotemporal diffusion patterns.

The paper is organized as follows. In Section 2 we describe the hydrodynamic model and the numerical approach to its solution. The solar radiation model is described in Section 3, while the ecological model and its coupling to hydrodynamics are presented in Section 4. The main features of the case study are introduced in Section 5 . The model is first validated with respect to its capability of reproducing realistic temperature fields. The results of several simulations of larval spread in Lake Garda are then presented and discussed. In Section 6 some conclusions are drawn on the applicability of the present approach to other similar problems and further developments of the present model are discussed.

\section{The hydrodynamic model and its numerical discretization}

The hydrodynamical model used in this work is based on the Reynolds averaged equations for 3D free-surface baroclinic flows, derived under hydrostatic and Boussinesq assumptions. Baroclinic terms allow the model to account for pressure variations due to fluid density variations, while a realistic definition of thermodynamic forcings enables the correct estimation of the heat budget of the water basin. The variations of fluid density responsible for the baroclinic component of fluid pressure are here assumed to be dependent on temperature only. Other factors (such as water salinity) have been disregarded, thus limiting the applicability of the model to freshwater ecosystems.

The equations of the hydrodynamic model read as follows:

$$
\begin{aligned}
& \frac{D u}{D t}=-g \frac{\partial \eta}{\partial x}-g \frac{\partial}{\partial x} \int_{z}^{\eta} \Delta \rho d z+\frac{\partial}{\partial z}\left(v_{V v} \frac{\partial u}{\partial z}\right)+f_{C} v, \\
& \frac{D v}{D t}=-g \frac{\partial \eta}{\partial y}-g \frac{\partial}{\partial y} \int_{z}^{\eta} \Delta \rho d z+\frac{\partial}{\partial z}\left(v_{V v} \frac{\partial v}{\partial z}\right)-f_{C} u, \\
& \frac{\partial \eta}{\partial t}=-\frac{\partial}{\partial x} \int_{-h}^{\eta} u d z-\frac{\partial}{\partial y} \int_{-h}^{\eta} v d z
\end{aligned}
$$

$$
\begin{aligned}
& \frac{D T}{D t}=\frac{\partial}{\partial z}\left(v_{T v} \frac{\partial T}{\partial z}\right)+q, \\
& \frac{\partial w}{\partial z}=-\left(\frac{\partial u}{\partial x}+\frac{\partial v}{\partial y}\right) .
\end{aligned}
$$

Flow velocities along $x, y$ and $z$ directions are denoted by $u, v$ and $w$, respectively. Water temperature and free surface elevations are denoted by $T$ and $\eta$, while $h, f_{C}$ and $q$ denote the bottom depth, Coriolis parameter and the sum of all heat sources and sinks (to be described later in greater detail). In the present formulation, density is computed by the state equation

$\rho=\rho_{0}\left[1-\alpha_{T}\left(T-T_{R}\right)^{2}\right]$,

where $T$ is the temperature expressed in ${ }^{\circ} \mathrm{C}, T_{R}=4{ }^{\circ} \mathrm{C}, \alpha_{T}=6.8 \times$ $10^{-6} \mathrm{~K}^{-2}$ and $\rho_{0}=1000 \mathrm{~kg} \mathrm{~m}^{-3}$ is a reference density value, while the relative density variations are denoted by $\Delta \rho=\left(\rho-\rho_{0}\right) / \rho_{0}$. Atmospheric pressure gradients have been neglected, since only applications to relatively small basins will be considered. It is to be remarked that the horizontal viscosity terms in the momentum and temperature equations have been omitted, since in the applications considered in this paper we have employed only relatively coarse meshes, for which the intrinsic numerical diffusion of the horizontal advection scheme is significant. In a more complete implementation, higher order interpolation methods should be used in the semi-Lagrangian scheme (see, e.g., Baudisch et al., 2006), along with an appropriate horizontal viscosity model. On the other hand, the algebraic eddy viscosity model derived in Colombini and Stocchino (2005) has been employed for the vertical viscosity terms.

From a technical perspective, the system of hydrodynamic equations (1)-(4) has been discretized by an extension of the semi-implicit and semi-Lagrangian numerical method proposed in Miglio et al. (1999), as described in Biotto (2007) in full details. A Crank-Nicolson time discretization has been chosen for the semi-implicit scheme. This method has in fact been proven to be linearly unconditionally stable with respect to the celerity of external gravity waves (Casulli and Cattani, 1994). The Coriolis terms have been discretized explicitly, since they do not imply severe stability restrictions on the computational meshes used in the present application. As for the spatial discretization, the $(x, y)$ plane has been covered with an unstructured triangular mesh, while the $z$ direction has been discretized in a suitable number of horizontal layers. Along the lines of Miglio et al. (1999), Raviart-Thomas elements of order 0 are used for the momentum equations, while $\mathbf{P}_{0}$ finite elements have been employed for the free surface and the temperature equations.

The temperature equation (4) is solved first, uncoupled from the momentum and free surface equations. As shown in (4), temperature advection is treated in a semi-Lagrangian fashion, employing respectively cubic and linear interpolations in the vertical and horizontal directions for the reconstruction step. The vertical turbulent viscosity term is discretized by a finite volume approach in space and by the Crank-Nicolson method in time. The computation of the heat fluxes due to solar radiation is described in detail in Section 3 . The solution of the temperature equation yields an updated density value that is used to compute baroclinic gradients. After this step, the algorithm follows exactly Miglio et al. (1999). The updated value of $\eta^{n+1}$ is computed by solving a Helmholtz equation obtained by substitution of (1) and (2) into (3). Finally, the velocity field is updated and the vertical velocity is recovered from the incompressibility constraint (5). It is to be remarked that, due to the lack of full coupling between baroclinic gradients and the momentum equation, the resulting time discretization is only conditionally stable with respect to internal gravity waves. On the other hand, unconditional stability with respect to external gravity waves is guaranteed 
by the semi-implicit discretization of the free surface equation. Noflow boundary conditions are imposed at the lateral boundaries of the spatial domain, while bottom friction and wind drag formulae are used at the bottom and free surface, respectively (Rodi, 1984).

\section{Solar radiation forcing on the water basin}

Modeling the heat fluxes at the surface of an enclosed water basin is essential to correctly reproduce the dynamics and the thermal budget of the basin itself. Furthermore, a correct estimation of water temperature is mandatory for the simulation of most biological processes. We will start by defining the various contributions to the heat flux at the surface of the water basin, while the modeling approach to the description of total heat sources within the basin will be discussed later on in this section. The total heat flux at the surface can be decomposed into shortwave radiation $Q_{O c}$, longwave radiation $Q_{O l}$, sensible heat exchange $Q_{l}$ and evaporation heat flux $Q_{e}$. Each of these terms will now be discussed in detail.

The total incoming shortwave radiation flux at the surface is defined according to Martin and McCutcheon (1999) as

$Q_{O c}=I_{0}\left(1-0.65 C_{n}^{2}\right)\left(1-R_{s}\right) a_{t}$,

where $I_{0}$ is the intensity of the radiation that would reach the Earth's surface in absence of the atmosphere, $C_{n}$ is a cloudiness dimensionless coefficient varying from 0 (clear sky) to 1 (completely cloudy sky), $a_{t}=0.6-0.7$ is the atmospheric attenuation term, which depends on soil reflectivity and on the content of water vapor in the atmosphere, and $R_{S}$ is the albedo coefficient. Both $I_{0}$ and $R_{S}$ are functions of the relative position with respect to the sun. Thus, they are time-dependent and have to be updated at each time step of the numerical simulation.

The albedo coefficient can be defined as $R_{S}=a(\alpha)^{-b}$ (Henderson-Sellers, 1986), where $\alpha$ is the angle (measured in degrees) of the sun position with respect to the ground, while $a$ and $b$ are two cloudiness-dependent coefficients defined as

\begin{tabular}{c|cc} 
& $a$ & $b$ \\
\hline$C_{n}<0.5$ & 2.2 & 0.97 \\
$C_{n}>0.5$ & 0.95 & 0.75
\end{tabular}

The incoming solar radiation at the surface is computed by taking into account the possible presence of mountains surrounding the lake, which implies that sun rays reach the water surface only when the sun inclination $\alpha$ is larger than a critical value $\alpha_{c r}$. Thus, one can define

$I_{0}= \begin{cases}0 & \alpha<\alpha_{c r}, \\ I_{S C} E_{0} \sin \alpha & \alpha>\alpha_{c r} .\end{cases}$

Here, $I_{S C}=1367 \mathrm{~W} \mathrm{~m}^{-2}$ is the value of the solar constant given in Fröhlich and Brusa (1981), while $E_{0}$ is a correction term that accounts for the eccentricity of the Earth's elliptic orbit. In Iqbal (1983), the approximate formula

$$
\begin{aligned}
E_{0}= & 1.00011+0.034221 \cos \Gamma+0.00128 \sin \Gamma \\
& +0.000719 \cos 2 \Gamma+0.000077 \sin 2 \Gamma,
\end{aligned}
$$

is proposed, where $\Gamma=2 \pi\left(d_{n}-1\right) / 365$ and $d_{n}$ denotes the progressive number corresponding to the current simulation day $\left(d_{n}=\right.$ 1 corresponding to January 1 and $d_{n}=365$ to December 31). Finally, the solar angle $\alpha$ is defined by the formula

$\sin \alpha=\sin \delta \sin \phi+\cos \delta \cos \phi \cos \omega$, where $\delta$ is the solar declination angle, defined as

$$
\begin{aligned}
\delta= & 0.006918-0.399912 \cos \Gamma+0.070257 \sin \Gamma \\
& -0.006758 \cos 2 \Gamma+0.000907 \sin 2 \Gamma \\
& -0.002697 \cos 3 \Gamma+0.00148 \sin 3 \Gamma
\end{aligned}
$$

$\phi$ is the geographic latitude and $\omega$ is an angular width varying linearly between $\omega_{a}=\operatorname{arcos}(-\tan \phi \tan \delta)$ and $-\omega_{a}$ in such a way that it is zero at noon.

As discussed in Imberger and Patterson (1981), the total longwave radiation $Q_{O l}$ is the difference between two contributions representing the incoming longwave radiation $Q_{O l}^{i}$ from the atmosphere to the water body and the outgoing longwave radiation $Q_{O l}^{o}$, so that $Q_{O l}=Q_{O l}^{i}-Q_{O l}^{o}$. These terms can be modeled as

$Q_{O l}^{o}=\varepsilon \sigma T_{S}^{4}$

and

$Q_{O l}^{i}=\varepsilon_{\mathrm{air}} \sigma\left(1+0.17 C_{n}^{2}\right) T_{\mathrm{air}}^{4}\left(1-R_{t}\right)$,

respectively. Here, $\varepsilon=0.972$ and $\varepsilon_{\text {air }}$ are water and air emissivities, respectively, $\sigma=5.669 \times 10^{-8} \mathrm{~W} \mathrm{~m}^{-2} \mathrm{~K}^{-4}$ is Stefan-Boltzmann constant, $T_{S}$ and $T_{\text {air }}$ are water surface and air temperatures, and $R_{t}=0.03$ is the value of the longwave reflection coefficient proposed in Henderson-Sellers (1986). Air emissivity can be computed by the formula $\varepsilon_{\text {air }}=C_{\varepsilon} T_{\text {air }}^{2}$ (Swinbank, 1963), where $C_{\varepsilon}=0.937 \times$ $10^{-5} \mathrm{~K}^{-2}$.

The radiative flux $Q_{e}$ due to evaporation is defined by the formula

$Q_{e}=\rho L_{w}\left(a_{w}+b_{w}|\mathbf{W}|\right)\left(e_{s}\left(T_{r}\right)-e_{s}\left(T_{s}\right)\right)$

proposed in Henderson-Sellers (1986), where $a_{w}=4.18 \times$ $10^{-11} \mathrm{~m} \mathrm{~s}^{-1} \mathrm{~Pa}^{-1}, b_{w}=0.95 \times 10^{-11} \mathrm{~Pa}^{-1}, L_{w}$ is the latent evaporation heat, $e_{s}$ is the saturated vapor pressure and $T_{r}$ the dewpoint temperature. For the latent evaporation heat and the saturated vapor pressure we use the formulae given in Henderson-Sellers (1986), i.e.,

$L_{w}=1000\left(2499-2.36 T_{s}^{\circ}\right)$,

$e_{S}\left(T_{S}^{\circ}\right)=2.171 \times 10^{10} e^{\left((-4157) /\left(T_{S}^{\circ}+239.09\right)\right)}$,

where $T_{S}^{\circ}$ is the water temperature at the surface expressed in Celsius.

The sensible heat flux $Q_{l}$ is modeled as proposed in Imberger and Patterson (1981):

$Q_{l}=C_{H} c_{\text {pair }} \rho_{\text {air }}|\mathbf{W}|\left(T_{\text {air }}-T_{S}\right)$,

where $C_{H}=1.4 \times 10^{-3}$ is the heat transfer coefficient at the height of $10 \mathrm{~m}$ above the surface, $\mathbf{W}$ is the wind velocity at the same height and $c_{\text {pair }}$ is the constant pressure specific heat of air.

Following Henderson-Sellers (1986), we assume that longwave radiation, sensible heat and evaporation fluxes at the surface have an impact on the superficial water layer only. Therefore, their values - along with surface shortwave flux - are used to impose a Neumann boundary condition at the surface for the temperature equation. Shortwave radiation, instead, can be assumed to penetrate to a larger extent into the water column, depending on the water turbidity. This effect is modeled in Henderson-Sellers (1986) as

$Q_{o c}(z)=Q_{o c}(0) \gamma e^{-\gamma(\eta-z)}, \quad z<\eta$

where $\gamma=1.1 z_{D S}^{-0.73}$ and $z_{D S}$ is the Secchi distance parameter, i.e., the smallest water depth at which a reference area disk is not visible from the surface. Thus, the total forcing term in the temperature 
equation for the interior of the water body (Eq. (4)) can be defined as

$q=\frac{1}{\rho c_{p}} \frac{\partial Q_{O c}}{\partial z}$,

where $c_{p}$ is the constant pressure specific heat.

\section{The ecological model and its coupling to hydrodynamics}

In this section we firstly discuss the features of the idealized species life cycle and derive a simple demographic model. Then, we introduce the procedures employed to evaluate larval transport. Finally, we describe the larval connectivity matrix, a tool that helps to analyze the core features of larval dispersal in a synthetic way.

\subsection{The species life cycle}

For the sake of simplicity, we limit our analysis to the case of semelparous species, i.e., to species whose individuals reproduce once in their lifetime and then die. We also assume that the reproductive season is as short as to be reasonably considered impulsive and that the sex ratio at birth and in the population is balanced. Although these hypotheses could seem too restrictive, they apply to a wide spectrum of populations and ecosystems, ranging from insects to mussels and from fish to plants (see, for instance, Pielou, 1977; Ricklefs and Miller, 1999). Finally, we consider species in which adults are sessile or highly sedentary (e.g., because they live anchored to the bottom) while propagules (either eggs or larvae) are benthonic (i.e., they can be transported by the waterflow). Note that this is the typical case of several mussel, coral and reef fish species (Guichard et al., 2004; Cowen and Sponaugle, 2009).

Our reference life cycle can thus be outlined as follows. Let $A_{t}$ be the density of adults at year $t$ in each location of the spatial domain. The density of adult females is $F_{t}=A_{t} / 2$, since we assume a balanced sex ratio. In each year $t$ adult females reproduce, generating eggs, then die. The abundance of female eggs is given by $E_{t}=f F_{t}$, where $f$ is the per capita net fertility (half of the total egg output). After a spawning event, the density of female larvae can be computed as $L_{t}=\sigma_{E} E_{t}$, where $\sigma_{E}$ represents the probability of survival from the egg to the larval stage. Then, larvae grow and are dispersed by the water flow. Let $\mathcal{L}_{t}$ be the density of larvae in each location of the spatial domain that are ready to settle after dispersal. The density of adult females in the next generation can thus be computed as $F_{t+1}=\sigma_{L}\left(\mathcal{L}_{t}\right) \mathcal{L}_{t}$, where $\sigma_{L}(\mathcal{L})$ is a density-dependent probability of survival from the larval to the adult stage. Density dependence has in fact important implications also in the context of larval recruitment (see, e.g., Cowen and Sponaugle, 2009 and more references therein). Finally, total population density can be computed from female density as $A_{t+1}=2 F_{t+1}$. Note that some of the steps just summarized describe yearly processes taking place at the local scale (i.e., adult reproduction and larval settlement), while others refer to phenomena that occur on a short time scale but on a large (basin) spatial scale (i.e., larval transport).

A number of models accounting for density dependence can be found in the literature (see, for instance, May, 1974; Hassell, 1975). Here we consider one of the simplest and most used, the Beverton-Holt model (Beverton and Holt, 1957), slightly modified as proposed in Hassell (1975) to account for demographic fluctuations. This model was originally formalized to describe the dynamics of fish stocks and has already been used to link larval dispersal to recruitment dynamics in sessile aquatic population with a benthonic life stage (e.g., James et al., 2002; Armsworth, 2002; Bode et al., 2006). In the modified Beverton-Holt model, larval survival is supposed to decrease with the abundance of settling larvae itself due to competition for limited resources (either nutrients or space, or both). From a mathematical perspective, we have

$\sigma_{L}(\mathcal{L})=\frac{\sigma_{0}}{(1+\beta \mathcal{L})^{\xi}}$,

where $\sigma_{0}$ is larval survival when the abundance of settling larvae is low, while $\beta$ and $\xi$ are two positive parameters describing the intensity of density dependence. For $\xi \leq 2$, the only nontrivial solution admitted by the model is a stationary equilibrium corresponding to the carrying capacity of the habitat. For $\xi>2$, the model can also display more complex dynamics, including periodic and chaotic fluctuations of population densities (Hassell, 1975).

\subsection{The larval transport model}

The local abundance of settling larvae after transport must be estimated on the basis of the velocity fields produced by the previously described hydrodynamic model. Larval transport by the water flow is modeled by a Lagrangian approach, which represents a common choice in the literature (e.g., Siegel et al., 2003; see also Werner et al., 2007; Cowen and Sponaugle, 2009 and more references therein), since it provides a natural and accurate framework to describe larval movement. In our simple approach, we consider that dispersing larvae are passively transported by currents, i.e., that they are unable to swim, orient themselves or perform vertical migrations, apart from the capability to settle at the bottom of the water body in the location where they reach maturity (e.g., Bode et al., 2006). Although the assumption of passive transport is quite common in the description of larval dispersal (e.g., James et al., 2002; Aiken et al., 2007), it should be noted that active movements can also play a remarkable role in determining the mean distance traveled by larvae (Steneck, 2006; Cowen et al., 2006; Paris et al., 2007; Werner et al., 2007; Cowen and Sponaugle, 2009).

The Lagrangian approach requires the computation of the trajectories followed by single larvae (individual based model). A system of three ordinary differential equations is integrated numerically to determine the position of each propagule. While in the horizontal directions the propagule velocity is identical to the velocity of the flow, the vertical velocity component $w_{p}$ is assumed to be given by the sum $w_{p}=w_{\text {flow }}+w_{\text {buoy }}$, where $w_{\text {flow }}$ is the flow velocity in the vertical direction and $w_{\text {buoy }}$ is the velocity induced by the buoyancy effect

$g \frac{\rho-\rho_{L}}{\rho_{L}}$,

where $g$ is the gravity constant, $\rho$ is water density and $\rho_{L}$ is the density of larvae. Other effects could be included in the evaluation of larval trajectories (see Miller, 2007, for a critical survey of the issues that do typically emerge in this context), but we do not explore this possibility here. In particular, we neglect the fluctuating components of larval velocity due to turbulence. This rather crude assumption is certainly not justified in general. However, in the applications considered in this paper, relatively coarse meshes have been used, for which the numerical diffusion of the horizontal advection scheme can be significant. We have verified the robustness of the results with respect to this hypothesis (see Section 5.3 below).

The computation of Lagrangian steps is accomplished by integrating the equations of motion for the propagules between 0 and a final time $t_{L}$, which represents the mean duration of the larval stage (assumed here to be constant; see, e.g., Bode et al., 2006; Aiken et al., 2007). Technically, numerical integration has been carried out by means of a standard explicit Euler method. To preserve accuracy we use a much smaller time step (generally $60 \mathrm{~s}$ ) than that used to solve the hydrodynamic model. As in Bode et al. (2006), it is assumed that at the end of the larval period propagules settle instantaneously in the element corresponding to their current posi- 
(a)

Year $t$

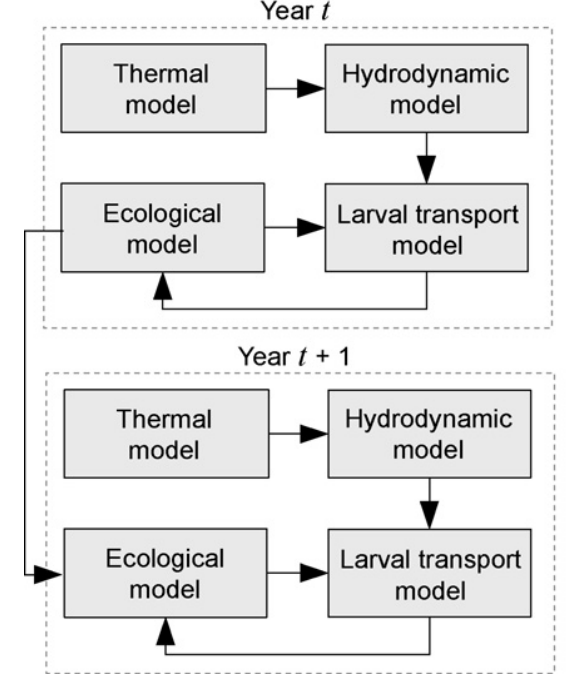

(b)

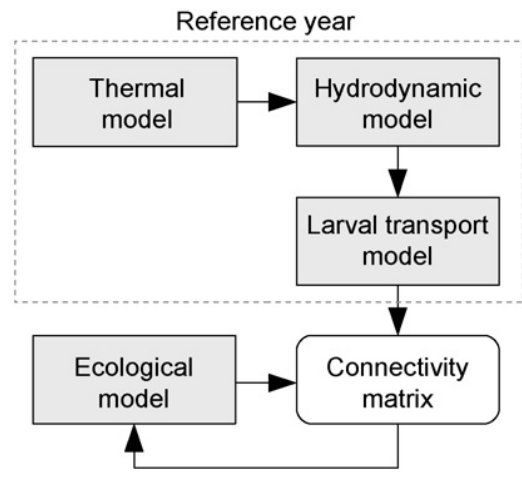

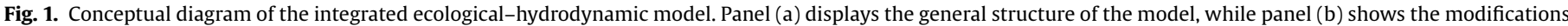
induced by the assumption of a reference year and the introduction of the larval connectivity matrix.

tion. Notice that not all the elements in the computational domain represent viable locations for the survival and growth of the population. Adverse conditions of illumination, temperature, pressure, flux velocity and/or substrate composition may prevent establishment (see, e.g., Cowen and Sponaugle, 2009). In the present work, we use depth and temperature as proxies for habitat viability. Therefore, we define a patch as viable if its mean depth is not greater than a threshold value $d_{\max }$ and its temperature during the spawning season is above $T_{\min }$. Larvae that settle in unviable patches are assumed to die instantaneously. Patches have been identified with the elements of the computational mesh used to solve numerically the equations of the hydrodynamic model. The problems that may arise if too coarse patches are used are discussed in Section 5.3.

\subsection{Simulation of long-term population dynamics}

Long-term spatiotemporal dynamics of population spread are obtained by suitably coupling the four components described so far, namely the thermal, hydrodynamic, ecological and larval transport models (Fig. 1(a)). First of all, the thermal and hydrodynamic modules have to be initialized. This could be done with the application of suitable data assimilation techniques. However, since this study is mainly aimed at demonstrating the feasibility of our integrated approach, and considering the small amount of available data for the case study analyzed in Section 5 , we have simply initialized the hydrodynamic model with a constant free surface value and zero velocity and run it for a sufficiently long start-up period (in the case considered in Section 5, one month) under realistic wind and solar forcing, so as to generate realistic hydrodynamical conditions. Thermal parameters are assumed to correspond to early summer, which represents the beginning of the spawning season in several freshwater species. Once a realistic circulation is established, the Lagrangian particles are released.

In the first year of simulation the ecological model is initialized by picking the point in the spatial domain where larvae (or adult individuals, which would additionally requires the evaluation of egg production through the ecological model) are first injected, while in the subsequent years the initialization is carried out reading stored data for adult abundance and computing the larval output due to a reproductive event. Then, for each active element (i.e., for each element with positive larval abundance) $n_{p}$ Lagrangian particles are released. The value of $n_{p}$ should be proportional to the total larval output in each element, but this could make model simulations very time-consuming. In fact, species with very high fecundities would require the tracking of huge numbers of particles. To avoid this unpleasant effect, the number $n_{p}$ of Lagrangian particles released in each active element is kept constant (we typically use $n_{p}=100$; see the relevant discussion in Section 5.3). As such, Lagrangian particles ultimately represent blobs of larvae whose abundance can be easily computed as the ratio between the total larval abundance of the native element and the number of released particles $n_{p}$. The initial position of each blob within an active elements is assigned assuming a uniform random distribution within the element in the two horizontal directions and within a $1 \mathrm{~m}$ layer at the bottom of the water column (to mimic spawning). Larval dispersal is then evaluated by means of the Lagrangian transport larval, while settlement and survival are finally evaluated with model (6) introduced above (see Fig. 1(a)).

This complex set of operations constitutes a year in the life cycle of the aquatic species. Therefore, it has to be repeated several times to study long-term spatiotemporal population dynamics. Since solar and wind forcing terms, although realistic, are indeed periodic and completely deterministic, a single run of the hydrodynamic component is sufficient to fully describe dispersal processes during a given year. If hydrodynamic and thermodynamic parameters are chosen with reference to a standard year and inter-annual variations of the environmental conditions are considered negligible, the results obtained for the reference year can then be extended to the years to come. In this work we assume that this simplified setting is acceptable for our case study. Indeed, the robustness analysis with respect to inter-annual variations described in Section 5.3 seems to justify our approach. This allows a significant reduction of the computational cost for a simulation that spans several species life cycles. For a given simulation scenario we thus perform a single numerical experiment, covering one spawning season, and derive the so-called larval connectivity matrix, which represents a powerful tool to understand the spatial relationships existing among patches (e.g., Armsworth, 2002; see also Cowen et al., 2006; Siegel et al., 2008).

Connectivity matrices are a standard tool in graph theory (Chartrand, 1985) and are widely used also in the ecological context to describe dispersal in fragmented habitats (Urban and Keitt, 2001). In particular, a connectivity matrix states which patches in the landscape are connected each other and what is the intensity 
of the connection. As such, the larval connectivity matrix $C$ has the following structure:

$C=\left[\begin{array}{cccc}c_{11} & c_{12} & \cdots & c_{1 n_{e l}} \\ c_{21} & \cdots & \cdots & \cdots \\ \vdots & \vdots & \vdots & \vdots \\ c_{n_{e l} 1} & \cdots & \cdots & c_{n_{e l} n_{e l}}\end{array}\right]$,

in which the element $c_{j i}$ represents the fraction of larvae that are generated in patch $i$ and settle in patch $j$ during one life cycle, while $n_{e l}$ is the number of patches used to cover the spatial domain on the $(x-y)$ plain. Larval transport can thus be evaluated in a given year $t$ as

$\mathcal{L}_{t}=\sigma_{L} C L_{t}$,

where $L_{t}$ and $\mathcal{L}_{t}$ represent larval abundance in each discrete location before and after transport, respectively. Once the larval connectivity matrix has been estimated, the simulation of long-term population is greatly simplified (Fig. 1(b)), just basically requiring the initialization of the ecological model, the computation of larval transport by means of the connectivity matrix and the evaluation of larval survival through the generalized Beverton-Holt model (6).

\section{A case study: the spread of a sedentary species in Lake Garda (Italy)}

In this section we present some results obtained applying the previously described model to a realistic case study, namely the spread of an ideal sedentary species in Lake Garda (Italy). We first introduce the domain of the case study and report some details about the validation of the hydrodynamic model with respect to its ability of reproducing realistic temperature fields. Then, we describe model simulations and analyze the impact of hydrodynamics on the spatial dynamics of the population. Finally, we investigate the robustness of the results and present a sensitivity analysis of the model outcomes with respect to variations of some important ecological and environmental parameters.

\subsection{Introduction to the case study area and model validation}

Lake Garda is the largest Italian lake. It lies at 45 degree of latitude, with an extension of about $368 \mathrm{~km}^{2}$ and a mean and maximum depth of $70 \mathrm{~m}$ and $350 \mathrm{~m}$, respectively (Fig. 2). To simulate the hydrodynamic model, we discretize the spatial domain with relatively coarse unstructured meshes with an approximate mesh size of $20 \mathrm{~m}$. As for the vertical discretization, we use layers with different thicknesses, ranging between $5 \mathrm{~m}$ (close to the water surface) and $50 \mathrm{~m}$ (at the bottom of the water body). However, this rather coarse spatial discretization is introduced only to reduce the computational cost in this first assessment of the performances of the integrated model, thus not representing an intrinsic limitation of our approach. In Biotto (2007), simulations with finer meshes were carried out for the hydrodynamic model only, along with a number of idealized baroclinic tests.

Here, we only present results with realistic environmental forcings. In particular, the effect of wind stress is incorporated into the model in a simple but realistic way, namely by considering a periodic wind forcing corresponding to the main two wind currents acting on the lake, the 'Peler' and 'Ora' winds. Peler wind blows southward on the upper portion of the lake from the early nighttime to late morning, approximately. It can reach a maximum speed of $15 \mathrm{~m} \mathrm{~s}^{-1}$. Ora wind blows in the opposite direction in the early afternoon, reaching a speed of approximately $12 \mathrm{~m} \mathrm{~s}^{-1}$.

The validation of the solar radiation model, together with its coupling to the hydrodynamic model, has been carried out by

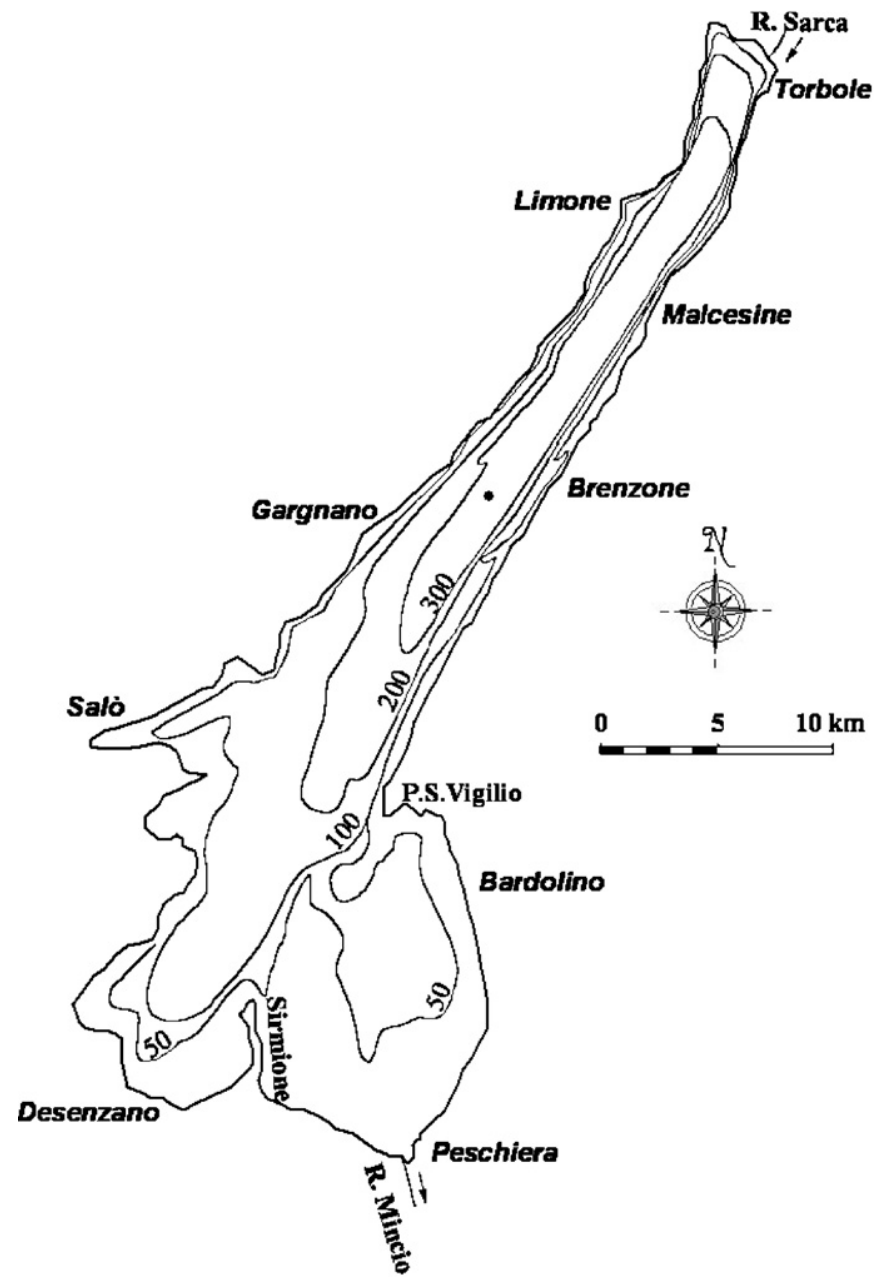

Fig. 2. Bathymetry of Lake Garda.

simulating one year of lake dynamics with typical solar radiation conditions. A simulation time step $\Delta t=900$ s has been used, while the time averaging parameter is set to $\vartheta=0.6$. The computed water temperature at the surface has been then averaged over the lake. Monthly time averages of this quantity have been compared to the corresponding climatological maximum and minimum average temperatures. Results are reported in Fig. 3, showing that the computed values are generally consistent with the climatologi-

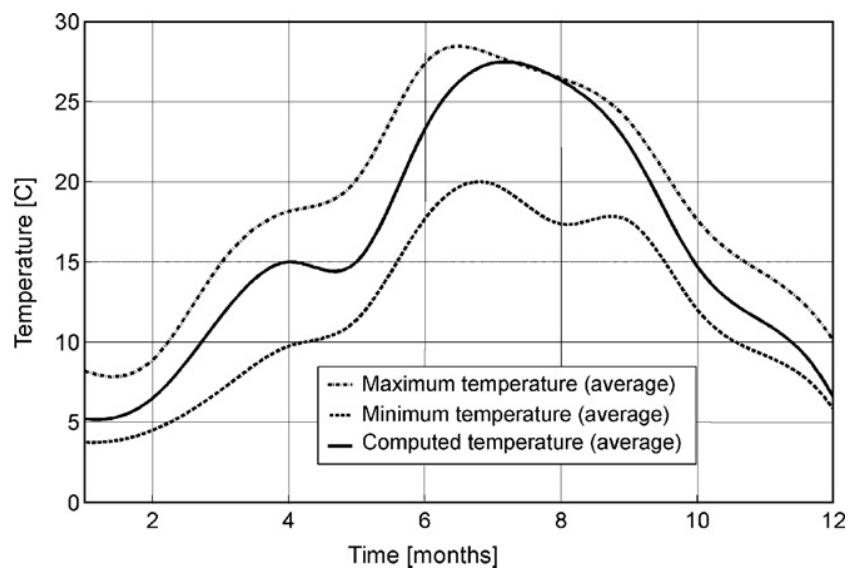

Fig. 3. Monthly averaged temperature obtained in a one-year-long simulation compared to climatological maximum and minimum temperatures. 
Table 1

Biological parameter values

\begin{tabular}{lcll}
\hline Parameter & Value & Parameter & Value \\
\hline$\sigma_{E}$ & 0.1 & $\rho_{L}$ & $\rho$ \\
$\sigma_{0}$ & 0.1 & $t_{L}$ & $7(\mathrm{~d})$ \\
$f$ & 1000 & $d_{\max }$ & $50(\mathrm{~m})$ \\
$\beta$ & 0.0001 & $T_{\min }$ & $0(\mathrm{C})$ \\
$\xi$ & 1 & & \\
\hline
\end{tabular}

cal ones, although a slight overestimation occurs during summer months.

A comparison with measured data has also been carried out for the temperature field at a single time instant. More specifically, in Fig. 4 the surface temperature on a typical September day is compared to the corresponding temperature field as recovered by NOAA satellite measurements (data available on the website of the University of Bern, Switzerland). Although it should be noted that the color maps used in the two plots are not exactly the same, the general pattern of the computed temperature field is in good agreement with the measured values.

\subsection{Simulation of species spread}

The computed velocity fields can be used to evaluate the effects of hydrodynamics on the spatial redistribution of larvae and generate the spatiotemporal patterns of species spread into the lake, namely by repeated application of the connectivity matrix as described by formula (7). To start a model simulation all the parameters have to be specified. In particular, hydrodynamic and thermodynamic parameters have been set to fit the case of Lake Garda as described above, while demographic parameters have been set to the values reported in Table 1 . Notice that $T_{\min }$ has been chosen so that temperature does not play a role in habitat viability, which is in this setting only dependent on depth. Furthermore, in this reference simulation the density of the larvae is taken to be equal to that of water.
Fig. 5 shows a typical example of model simulation. We assume that the species begins its spread close to Peschiera sul Garda (approximately marked by a black arrow in panel (a) of Fig. 5). In a few years it reaches the whole south basin of Lake Garda. For the parameter setting of Fig. 5, population density in each patch settles on a stationary value. This is obviously due to the introduction of a compensatory density dependence $(\xi=1)$. However, we observe that the population is not homogeneously distributed among all the viable patches of the lake. Specifically, the carrying capacity of some site cannot be completely exploited due to recruitment limitations, i.e., because there are less settling larvae than larvae that are potentially allowed to settle. As already noticed by Armsworth (2002), both density dependence and recruitment limitation can in fact play a role in determining population dynamics. Our analysis shows in fact that demographic processes and transport phenomena due to hydrodynamics are interlinked and work together to determine the evolution of spatiotemporal population patterns. As such, they should always be analyzed together in long-term studies.

Experiments like that described in Fig. 5 can help answering some of the basic questions set in Section 1, namely where do larvae go, or where do they come from (Levin, 2006). Key to the understanding of such questions is the larval connectivity matrix $C$ (Fig. 6(a)). In fact, by definition, the $i$ th column of $C$ determines what is the fraction of larvae starting from patch $i$ and arriving in patch $j$. In the same way, it is also possible to estimate where do larvae come from by just reading the rows of $C$. It may help to display the connectivity matrix in a spatially explicit setting. Fig. 6(b) shows the strongest connections among patches defined by matrix $C\left(c_{i j}>0.05\right.$, corresponding to the black dots in the sparsity plot reported in panel (a)). Interestingly, relatively few patches are characterized by several of such connections, while most patches seem to be poorly connected. Also, it turns out that the strongest links between patches occur among rather distant locations.

The larval connectivity matrix can be also used to evaluate the so-called dispersal kernel (Turchin, 1998), which states what is

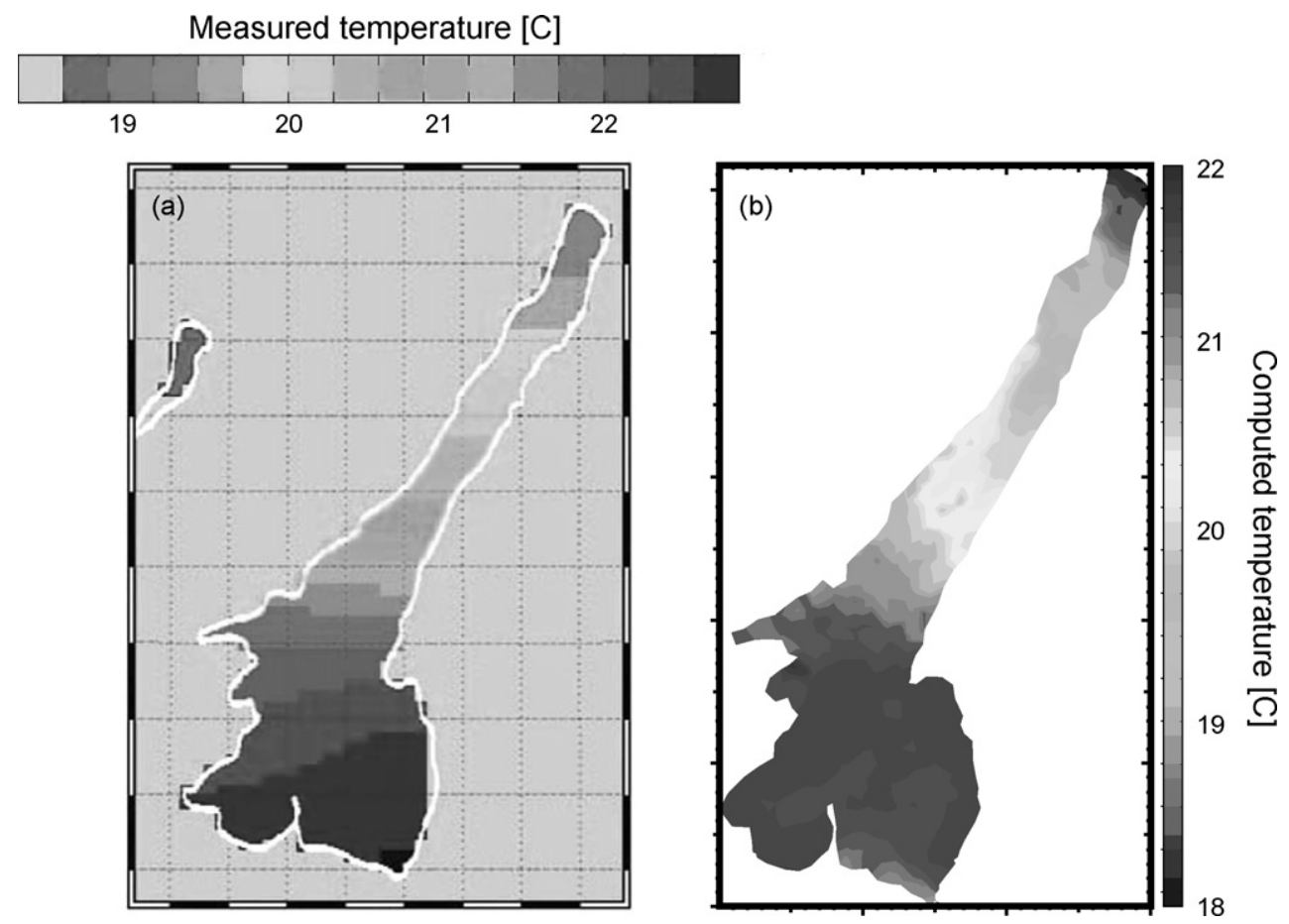

Fig. 4. Water temperature at the surface in a typical September day: (a) remote sensing measurement from NOAA satellite; (b) numerically simulated temperature. 

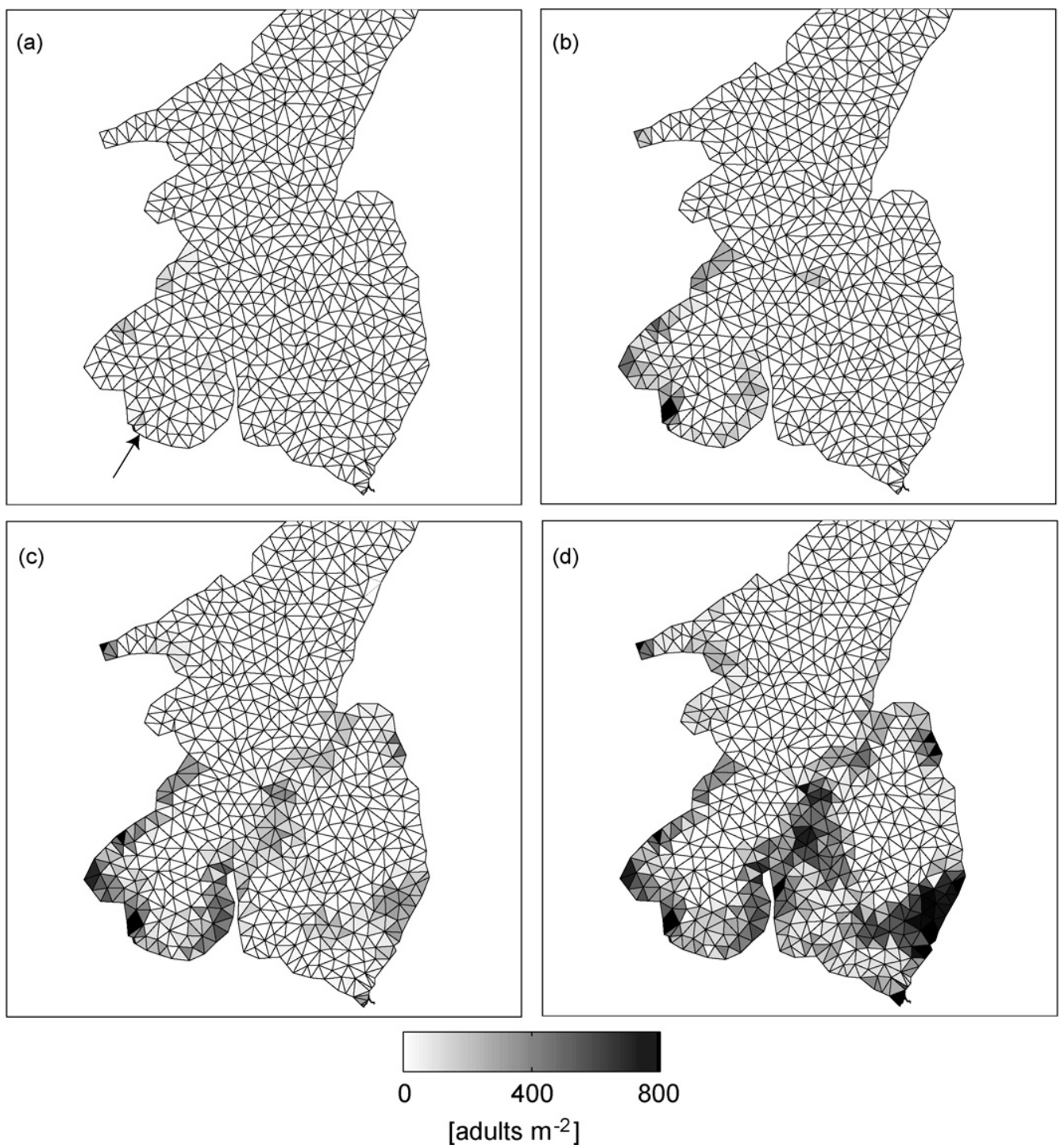

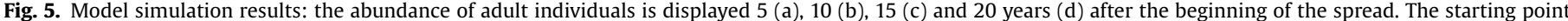

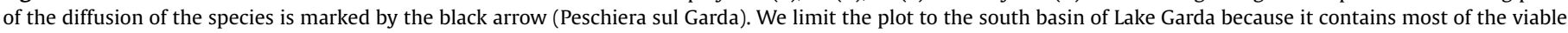
patches. Model parameters are as in Table 1.

the fraction of propagules that is expected to travel a given distance from the release point as a function of the distance from the native patch itself. Although dispersal kernels are usually rigorously defined for isotropic environments (in which dispersal is not affected by spatial dishomogeneity), they are often estimated also in cases in which the hypothesis of spatial homogeneity is violated, as in the case of larval transport (Siegel et al., 2003; Steneck, 2006). Fig. 6(c) displays the mean dispersal kernel (i.e., the average of the dispersal kernels estimated with reference to each viable site in Lake Garda) for the scenario depicted in Fig. 5. The inset shows the dispersal kernel obtained by excluding the effects of self-recruitment, i.e., by excluding larvae that settle in the same patch in which they have been released. As such, the kernel in the inset describes the probability that a propagule travels a given distance, provided that it leaves the native patch. Three regions can be identified in the dispersal kernel, respectively corresponding to short-, medium- and long-distance dispersal. Quite interestingly, more than $40 \%$ of the larvae do not move beyond $1 \mathrm{~km}$ (10\% excluding self-recruitment) from the native element (shortdistance dispersal), while more than $9 \%$ (13\% if self-recruitment is disregarded) are dispersed for more than $10 \mathrm{~km}$ (long-distance dis- persal), approximately three times the distance traveled by larvae on average (about $3629 \mathrm{~m}$ ). Note that such long-distance dispersal events may be very important in the maintenance of the population at the basin scale (Cowen et al., 2000, 2003, 2006; Kraft et al., 2002).

The larval dispersal kernel is a very useful tool, since it helps summarizing the core characteristics of larval dispersal in the basin. However, some features of larval dispersal are highly sitedependent. In particular, both self-recruitment (i.e., the local retention of larvae in their native patch, corresponding to the main diagonal of $C$; Fig. 6, panel (d)) and the mean distance traveled by larvae (e) vary remarkably among patches (as found in empirical observations; e.g., Cowen et al., 2006; Cowen and Sponaugle, 2009). The importance of self-recruitment represents another highly debated topic in the literature on larval dispersal (Cowen et al., 2000, 2006; Levin, 2006; Becker et al., 2007). Simple statistical analyses show that in the scenario of Fig. 5 the mean fraction of larvae being locally retained in each viable element is about $31.27 \%$ (variance $12.06 \%$ ). We remark that estimating local larval retention can give important cues on the planning of management policies (e.g., in the case of invasive alien species or marine pro- 

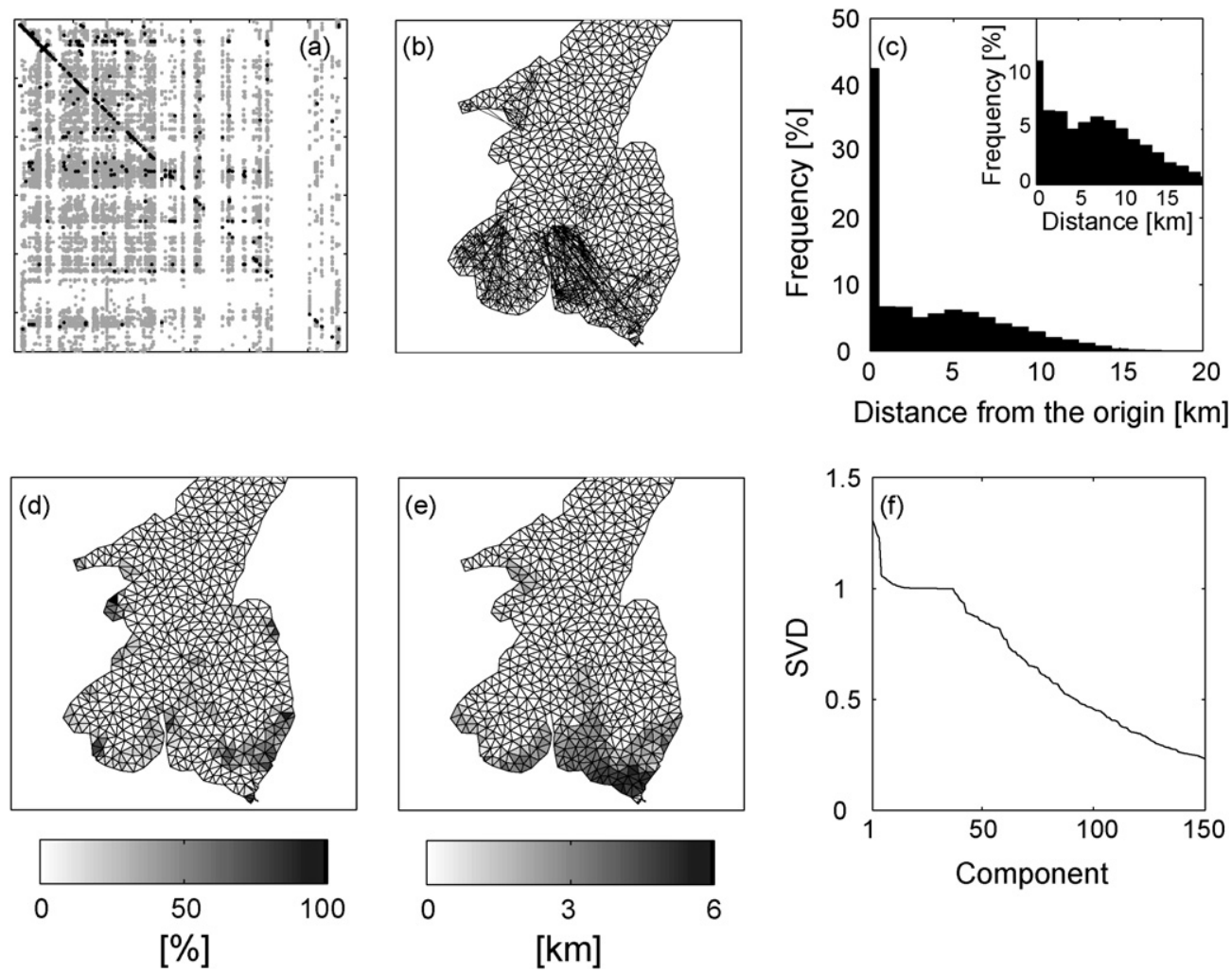

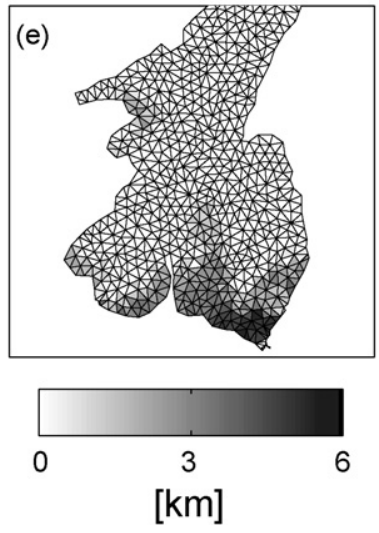

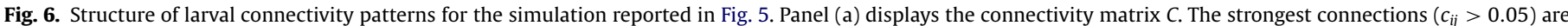

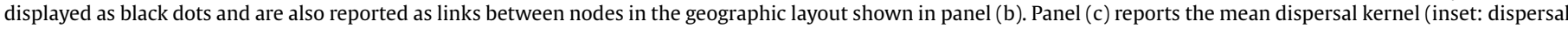

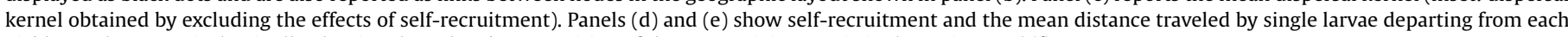
viable patch, respectively. Finally, the singular value decomposition of the connectivity matrix is shown in panel (f).

tected areas; e.g., Guichard et al., 2004; Werner et al., 2007; Cowen and Sponaugle, 2009), specifically suggesting an a priori estimation of the potential effectiveness of control actions planned at the local/basin scale. A visual inspection of Fig. 6(d) and (e) shows that patches with low self-recruitment are typically characterized by short mean dispersal distances, and viceversa, with some notable exceptions close to the south-eastern coasts of Lake Garda. We also remark that contrasting panels (d) and (e) of Fig. 6 to panel (b) yields a better understanding of the structure of larval connectivity patterns, specifically defining larval sources and sinks (Bode et al., 2006).

We finally note that sometimes the visual inspection of the spatiotemporal patterns is not sufficient for a precise assessment of larval connectivity patterns. Various methods based on the analysis of the connectivity matrix have been proposed (e.g., Cowen and Sponaugle, 2009; Siegel et al., 2008). In this work, we consider the singular values of the connectivity matrix (see, e.g., Golub and Van Loan, 1989) as a synthetic indicator of its characteristics. In case of the reference simulation described above, the singular values are displayed in Fig. 6(f). This tool is also used in Section 5.4 to estimate the impact of different simulation scenarios on the spatiotemporal evolution of species spread. A general result is that singular values of the connectivity matrix decay quite slowly, as displayed in Fig. 6(f), so that connectivity patterns cannot be explained on the basis of few principal components of the matrix. As a consequence, as it will be discussed in greater detail in the next section, it is unlikely that simplified box models could be successfully applied to analyze the problem at hand. This finding seems to support the view that sufficiently high resolution models are required to fully understand spatiotemporal population dynamics in basins like Lake Garda.

\subsection{Robustness of results with respect to modeling assumptions}

It should be always kept in mind that modeling assumptions can remarkably impact the outcomes of model simulations. Therefore, before presenting a sensitivity analysis regarding the role of ecological and thermodynamical parameters, it is worth assessing the influence of some crucial modeling hypotheses on the results presented so far.

As a first example, the spatial resolution at which the model analysis is carried out can have a significant impact on model outcomes. In our analyses, we have used a grid of 1593 elements and about 20 vertical layers with thickness varying between $5 \mathrm{~m}$ (close to the surface) and $50 \mathrm{~m}$ (close to the bottom). This vertical discretization turned out to be an acceptable trade-off between the accuracy of the results and the efficiency of the computational scheme. Simulations with a coarser vertical discretization lead in fact to inaccuracies in the computation of velocity fields. Parameter values such as the number $n_{p}$ of Lagrangian particles released from each active element can also be very important for the accuracy of the results. With this respect, we have repeated model runs using $n_{p}=50,150,200$. Very similar results were observed for $n_{p}=100,150,200$, while the use of $n_{p}=50$ seemed to result in significant underestimation of the species spread with respect to the simulations employing more particles.

Another characteristic feature of our approach is that the patches used to build the connectivity matrix correspond to the triangular elements resulting from the spatial discretization of the domain. In contrast, in most real applications, the number of patches used to analyze connectivity is rather small (e.g., Galindo et al., 2006; Becker et al., 2007; Planes et al., 2009). Therefore, in order to 
understand the sensitivity of the presented results with respect to different patch sizes, we rearranged the information used to build the reference adjacency matrix so as to describe the connectivity structure of a (smaller) pre-defined number of rectangular patches, obtained by regularly partitioning the spatial domain of the case study. The resulting population patterns seem to show that a coarse partitioning induces a severe misrepresentation of the spread process (Fig. 7(a) and (b)). This is consistent with the slow decay of the singular values of the connectivity matrix that is observed when small patches are used (Fig. 6(f)).

While describing the Lagrangian approach followed to model propagule dispersal, we have completely neglected the turbulent component of the velocity field experienced by larvae. To explicitly test the robustness of our results with respect to this assumption we have performed some additional numerical experiments in which we have introduced a simple stochastic model for turbulent larval
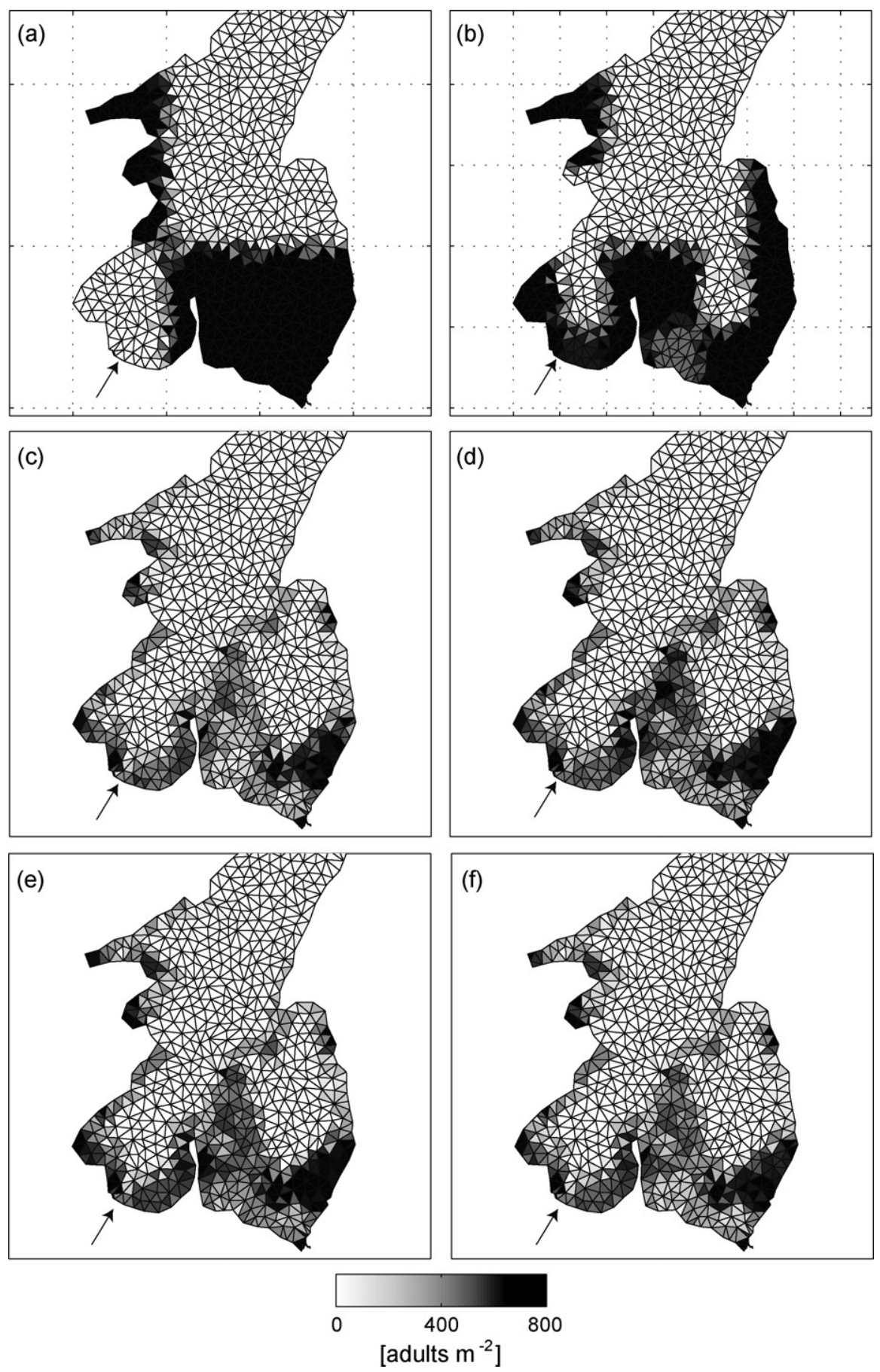

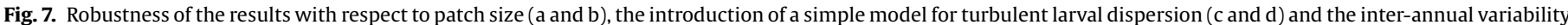

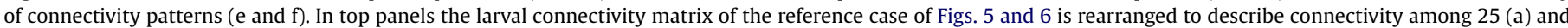

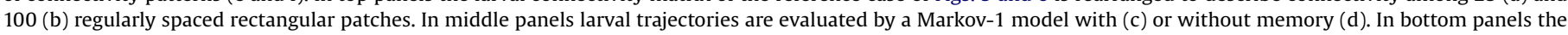

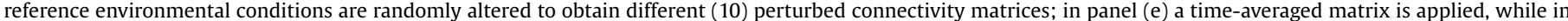

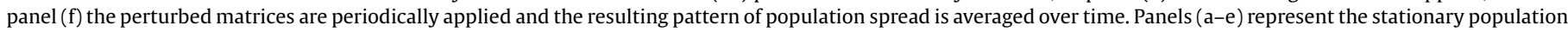

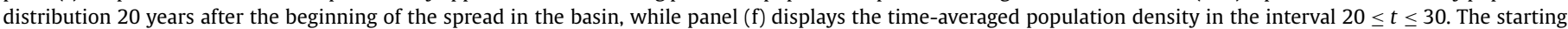

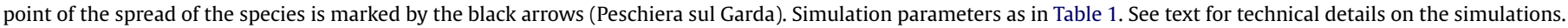


dispersion. Specifically, we have used a Markov-1 model (Berloff and McWilliams, 2002), which represents one of the most common choices in the analysis of larval dispersal patterns in marine ecosystems (e.g., Siegel et al., 2003; Paris et al., 2007) and considered two distinct cases: in the first one we assumed that the random process has no memory (Fig. 7(c)), while in the second case the temporal evolution of the turbulent component of larval velocity is characterized by some degree of temporal correlation (Fig. 7(d)). In both cases we parameterized the model as suggested by Dewey and Stegen (1995) and fixed the ratio between the turbulent and the mean kinetic energy to 0.05 , which is a typical value for ocean flows (see again Dewey and Stegen, 1995), thus probably representing a slight overestimation for our case study. The simulations displayed in the middle panels of Fig. 7 confirm that our choice of neglecting the fluctuating components of the turbulent flow has indeed little impact on long-term spatiotemporal patterns of population spread, given the numerical diffusion introduced by the applied numerical scheme.

In the reference simulation displayed in Fig. 5 we have assumed that our ideal species begins its spread close to Peschiera sul Garda, which is one of the most important towns located on the shores of Lake Garda. If we consider the diffusion of a newly introduced alien species, it seems completely reasonable to assume that the spread could start from a city like Peschiera. However, this is just a working hypothesis. We have thus performed several model simulations in which we altered the starting point of the spread process and found that this feature has a remarkable impact on the spatiotemporal population dynamics only in the short run. In fact, simulations starting from different initial conditions seem to converge in the long
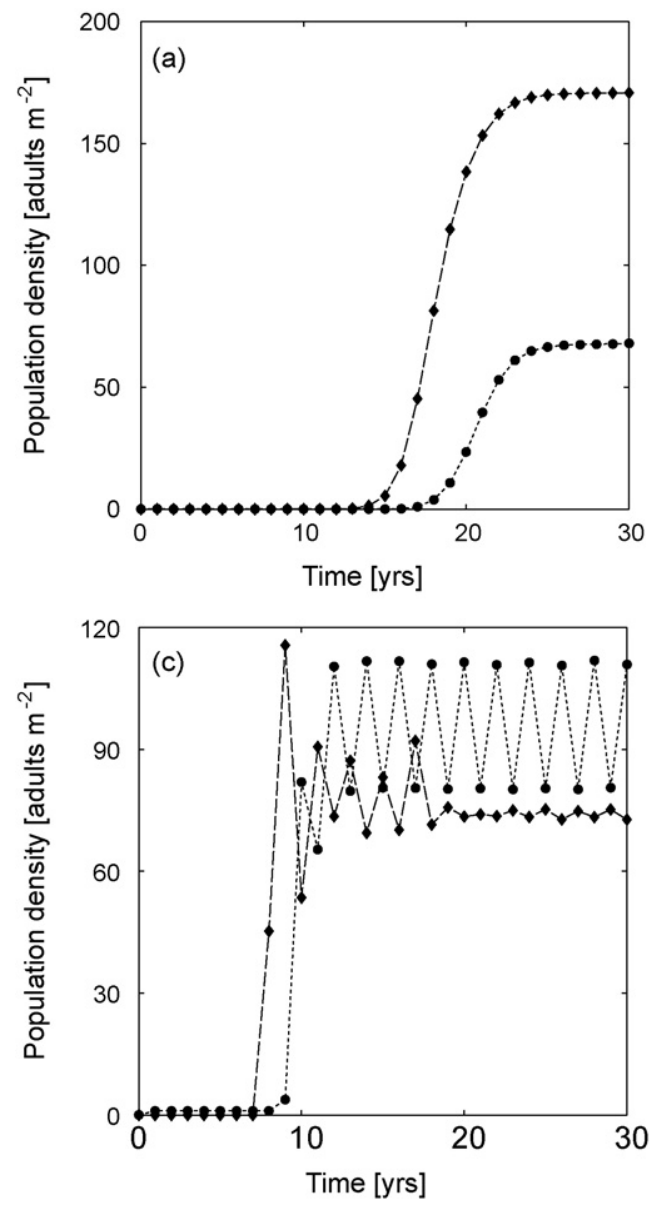

run to the same stationary spatial pattern of population density, at least for $\xi \leq 2$.

Concerning the description of the physical forcings acting on the water body, a key aspect of our modeling approach is the assumption of reference environmental conditions. In order to understand the extent to which this assumption is correct, we have run the hydrodynamic model for 10 different spawing periods with environmental conditions differing from those of the reference year. Such conditions have been obtained adding stochastic perturbations of the baseline temperature, cloudiness and wind fields, and by randomly altering the starting day of the larval period. The ecological model has then been run for a 20 years period in which two cyles of 10 years employing the previously obtained velocity fields were repeated. The resulting analysis (Fig. 7(e) and (f)) shows that using either the perturbed matrices or an averaged connectivity matrix does not produce remarkable impacts on the spatiotemporal density patterns at the basin scale, although altering connectivity can ease recruitment limitation in the southern shores of the basin. Therefore, we conclude that our simplifying assumption of a reference year is indeed acceptable for our purposes.

\subsection{Sensitivity analysis}

The results of model simulations do obviously depend upon the specific parameter setting considered. Therefore, it is important to perform a sensitivity analysis of the model outcomes with respect to changes in the parameter values.

Concerning the ecological model, we have investigated the role of the demographic parameters $\sigma_{0}$, which quantifies survival from
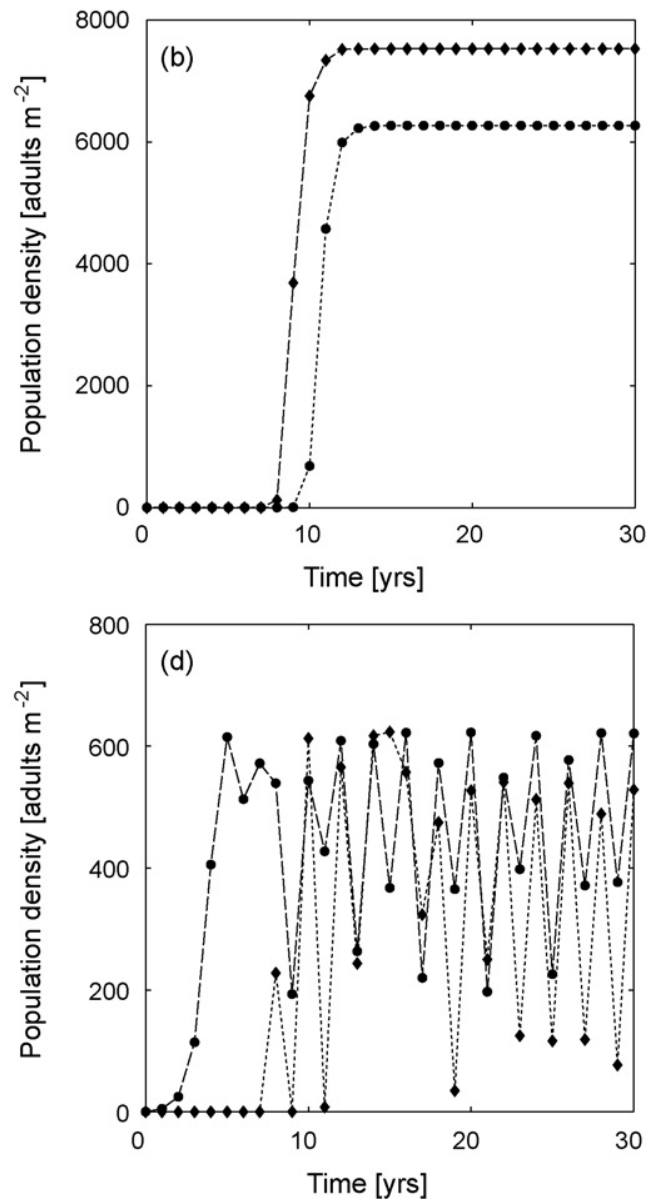

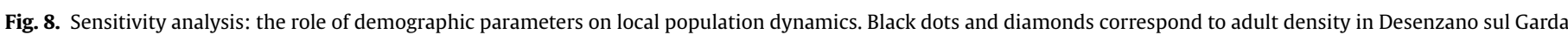

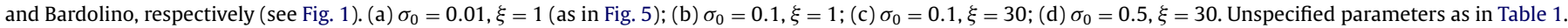


the larval stage to the adult phase (i.e., survival during transport and after settlement), and $\xi$, which describes the intensity of density dependence. Demographic parameters can have a strong impact on the densities reached by local colonies. For instance, higher values of $\sigma_{0}$ obviously lead to more abundant local populations (panels (a) and (b) of Fig. 8). As already remarked above, the densities reached by local colonies are highly site-dependent. As an example, population densities in Desenzano sul Garda (dots) are much lower than those recorded in Bardolino (diamonds). However, different values of the biological parameters $\sigma_{0}$ and $\xi$ cannot alter the structure of the larval connectivity matrix $C$. Therefore, the long-term spatial pattern of species spreading remains qualitatively unaltered. On the other hand, increasing values of either $\sigma_{0}$ or $\xi$ (or both) lead to interesting outcomes at the local scale. Local populations may in fact display wide demographic fluctuations. Such fluctuations can be either regular (periodic dynamics, Fig. 8(c)) or highly irregular (chaotic dynamics, Fig. 8(d)) and result from the introduction of a strong overcompensatory density dependence. Therefore, this typical feature of the modified Beverton-Holt model (6) is still preserved when ecology and hydrodynamics are coupled. Quite interestingly, we observe that different spatial locations are in general characterized by different population densities averaged over time. Specifically, mean population densities in Desenzano sul Garda are higher than those in Bardolino in case of periodic dynamics (Fig. 8(c)). Also, notice that demographic fluctuations under periodic regime can be characterized by very different oscillation amplitudes and can be desynchronized (Earn et al., 2000; Earn and Levin, 2006). Years with high population densities in Desenzano sul Garda correspond in fact to years with low densities in Bardolino.

On the other hand, some other biological parameters may influence the structure of the connectivity matrix $C$. This is the case, for instance, of larval density $\rho_{L}$. This result should be obviously expected: in fact, if $\rho_{L}$ is greater than water density, then the vertical acceleration due to gravity becomes active and forces larvae towards the floor of the lake. As a result, propagules travel shorter distances than in the case in which $\rho_{L}=\rho$. However, this does not necessarily imply negative impacts on the spread of the species. Top panels of Fig. 9 show what is expected to happen for small positive variations of larval density (on the order of $0.1 \%$ of water density). Although the mean distance traveled by dispersing larvae turns out to be shorter than in the previously analyzed scenario $(2902 \mathrm{~m})$, the spread of the species can be even promoted. Notice in fact that in this simulation scenario the species is expected to establish with high population densities also along the southern coasts of Lake Garda. This apparent paradox can be explained by noting that, due to spatial correlation, the closer a larva is dispersed from a viable patch, the higher the probability it can settle in a viable patch. Small positive variations of larval densities may thus mitigate recruitment limitations in otherwise poorly connected viable sites. However, larger values of $\rho_{L}$ do obviously reduce the spread of the species in a remarkable way. As an example, bottom panels of Fig. 9 report the results of a simulation obtained with a larger positive variations of
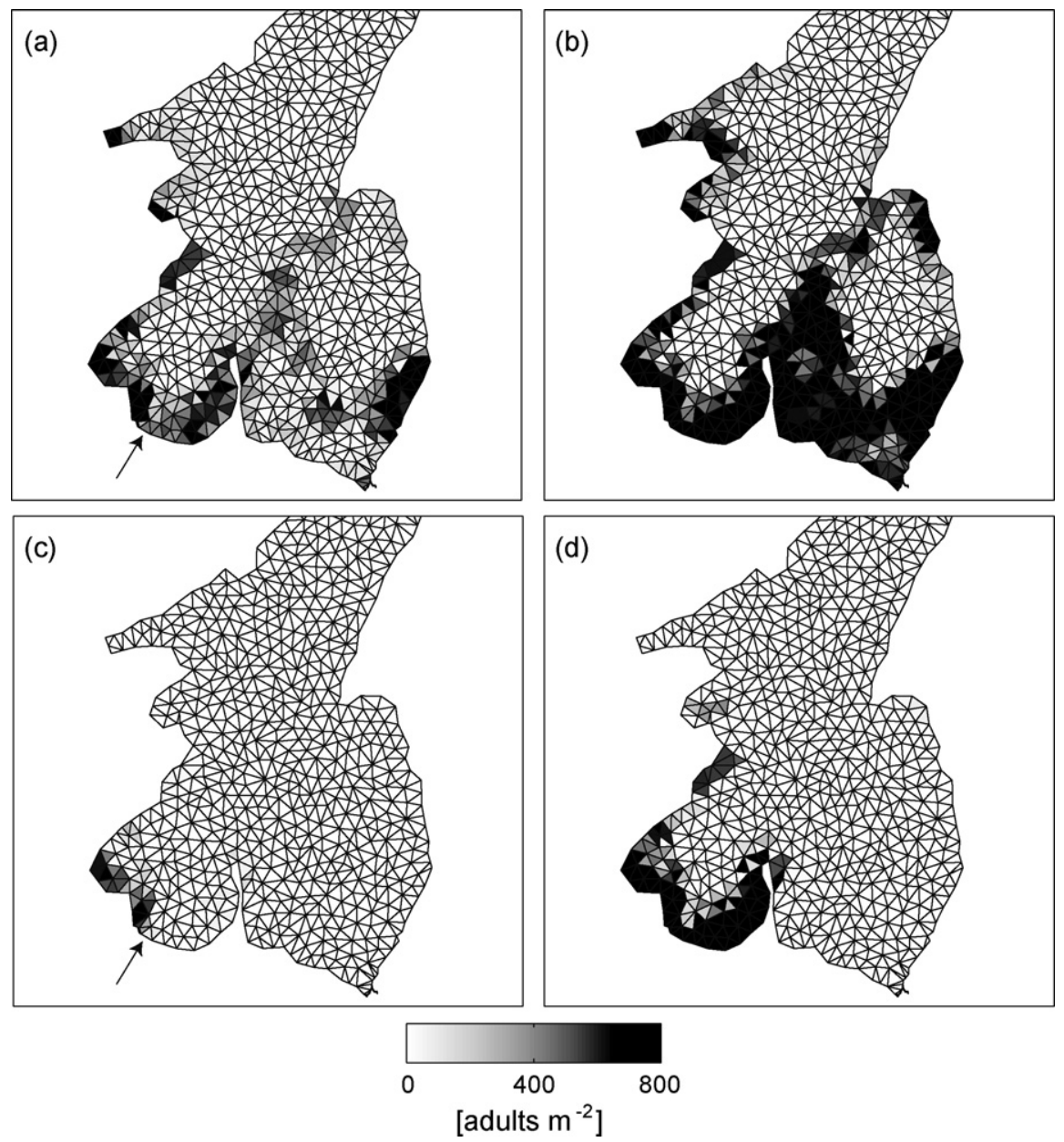

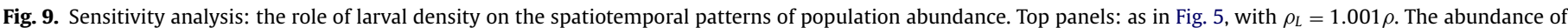

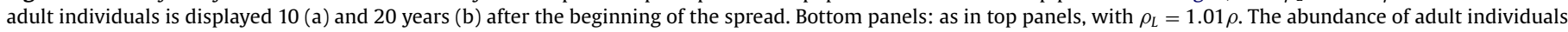

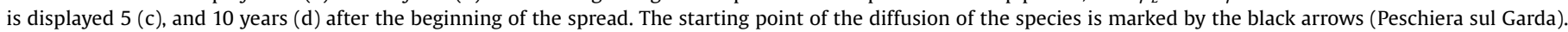
Other simulation parameters as in Table 1. 

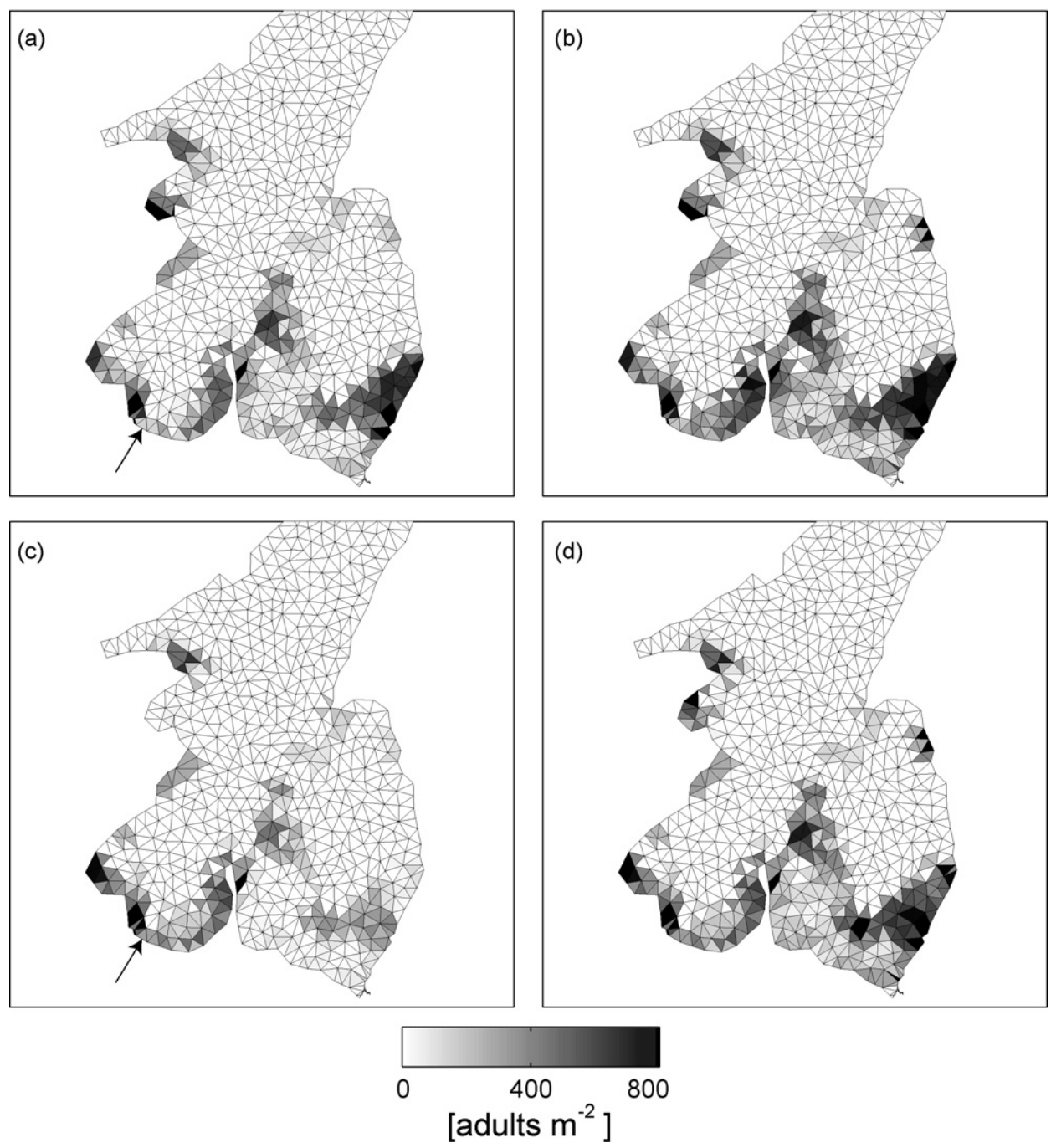

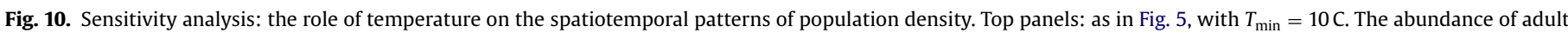

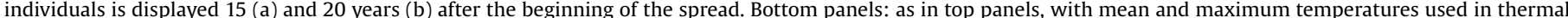

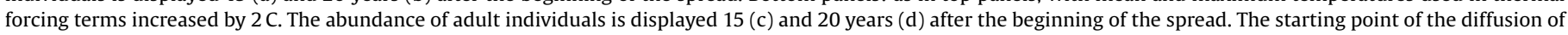
the species is marked by the black arrows (Peschiera sul Garda). Other parameters as in Table 1.

larval density with respect to water density (on the order of $1 \%$ ). In this case, larvae travel remarkably shorter distances ( $995 \mathrm{~m}$ ) and the spread of the species is limited to the south-western part of the lake, close to the starting point of the species diffusion.

Environmental parameters can have a sensible impact on longterm population dynamics. For instance, water temperature plays an important role in the spread of aquatic species in several ways (see, e.g., Cowen and Sponaugle, 2009 and references therein). For instance, spawning is often regulated by temperature, meaning that the release of propagules cannot happen below a given temperature threshold. Also the duration of the larval phase may depend on temperature, as well as other demographic parameters such as larval survival or adult fertility. Top panels of Fig. 10 show a model simulation in which the aquatic species is supposed to be sensitive to temperature. Specifically, we assume that a patch is viable for the population only if the mean temperature during the spawning season exceeds a minimum value $T_{\min }=10^{\circ} \mathrm{C}$ and the mean depth does not exceed $d_{\max }=50 \mathrm{~m}$, as in previous model runs. This assumption does obviously produce a change in larval connectivity patterns. A comparison between the top panels of Fig. 10 and the bottom panels of Fig. 5 shows that, although the stationary population distribution turns out to be quite similar in the two simulation scenarios, transient spatiotemporal dynamics can be different, mainly due to density dependence. A decrease in the number of viable patches leads in fact to a smaller abundance of both released and settling larvae, which then experience higher survival rates, thus promoting a faster population growth. Temperature has also a direct influence on water flows and currents. As such, variations of mean and maximum temperature values used in thermal forcing terms can modify water circulation in the water body, thus altering larval redistribution patterns. As an example, bottom panels of Fig. 10 show what happens in a scenario in which mean and maximum temperatures used in thermal forcing terms are $2^{\circ} \mathrm{C}$ higher. As already recorded in the previous simulations, the interplay between density-dependent demographic dynamics and hydrodynamics may produce nontrivial effects on the spatiotemporal evolution of the spread. This suggests that climate change may have a remarkable influence on spatiotemporal invasion patterns (Fields et al., 1993; Roy et al., 2001; Cowen and Sponaugle, 2009). We have also found that other environmental forcings may have less noticeable effects on long-term population patterns. For instance, deviations of wind speed intensities in the order of $25 \%$ from the reference mean values do not produce remarkable differences in the structure of larval connectivity.

Finally, the singular values of the connectivity matrices obtained for some of the parametric scenarios introduced in this section are 


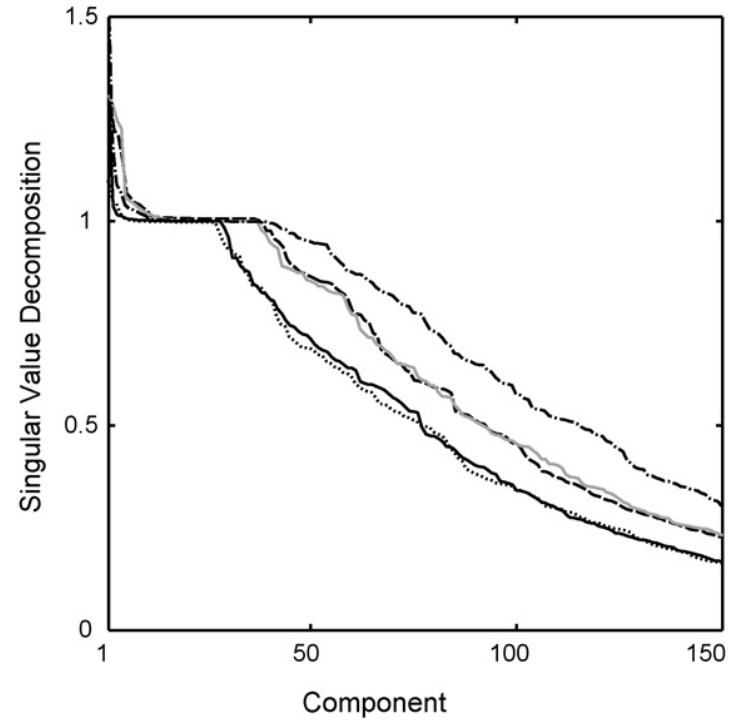

Fig. 11. First 150 singular values of the connectivity matrices computed in the cases of $\rho_{L}=1.001 \rho$ (Fig. 9(a) and (b), dash-dotted line), $T_{\min }=10^{\circ} \mathrm{C}$ (Fig. 10(a) and (b), black solid line), $T_{\min }=10^{\circ} \mathrm{C}$ with mean and maximum temperature used in thermal forcings increased by $2 \mathrm{C}$ (Fig. 10(c) and (d), dotted line), mean wind intensity in the order of $75 \%$ of the reference value (dashed line). The first 150 singular values of the connectivity matrix of the reference case are also reported (gray solid line). See text for details.

displayed in Fig. 11. We note that both larval density (dash-dotted line) and sensitivity to temperature (black solid line) have a remarkable impact on the structure of larval connectivity (the gray solid line represents the singular values of the baseline case, as already reported in Fig. 6(f)), even if they do not change water flows in Lake Garda. On the contrary, small variations of thermal (dotted line) or wind forcings (dashed line) produce less notable effects on the structure of the connectivity matrix.

\section{Conclusions}

In this work we have shown how a simple ecological model for the local demographic dynamics of a sedentary aquatic species can be coupled to a realistic description of the transport effects at the basin scale, in order to study the long-term dynamics of the population in a closed, thermally forced water body. Studying the evolution of these spatiotemporal patterns is a very complex task, which requires an integrated and highly interdisciplinary approach. However, understanding the patterns of species spread is mandatory in both conservation and management problems.

We have described local demographic dynamics by means of a modified Beverton-Holt model. The underlying hydrodynamic model implements a semi-implicit and semi-Lagrangian method, thus ensuring efficiency and accuracy. Moreover, the introduction of realistic thermal and environmental forcings (solar radiation, wind stress, etc.) has allowed to link the hydrodynamics to a range of key biological processes. A Lagrangian approach has been used for evaluating larval trajectories. In addition, under some reasonable simplifying hypotheses, less costly long-term model simulations have been performed by estimating a so-called larval connectivity matrix, which allowed off-line computation of hydrodynamical effects on larval circulation. While analyzing a realistic case study (the spread of an ideal sedentary aquatic species in Lake Garda, Italy) we have found that, although transport and retention effects are extremely site-dependent, larvae can generally travel over relatively long distances. Long-distance dispersal accounts in fact for about $10 \%$ of total dispersal, thus possibly representing a key feature for the definition of the population dynamics at the basin scale. A sensitivity analysis of the model has shown that both biological parameters and thermal forcing can play a major role in determining long-term demographic dynamics, as well as the spatial distribution of the population.

Future developments of this work will concern an improvement of the accuracy of the numerical and turbulence models employed, the use of more realistic ecological models, a more thorough validation of the hydrodynamic component and the use of higher spatial resolutions. Also, the description of larval transport can be made more accurate by introducing some realistic details such as the possibility of swimming or performing oriented vertical migrations. All these improvements should be then validated against field data with the aim of producing an accurate (yet agile) model for analyzing complex spread problems in aquatic ecosystems.

\section{Acknowledgments}

The authors thank Edie Miglio, Marino Gatto, Renato Casagrandi (Politecnico di Milano) and Giulio de Leo (Università degli Studi di Parma) for useful comments and hints. We also thank two anonymous reviewers for useful criticisms that helped us to improve the quality of our manuscript. A first version of the solar radiation model was developed by Dr. Giuliano Rizzi in his Master Thesis in Environmental Engineering (Università degli Studi di Trento, 1998). Finally, an essential input for this work was the encouragement and support we all received from Fausto Saleri.

\section{References}

Aiken, C.M., Navarrete, S.A., Castillo, M.I., Castilla, J.C., 2007. Along-shore larval dispersal kernels in a numerical ocean model of the central Chilean coast. Marine Ecology Progress Series 339, 13-24.

Armsworth, P.R., 2002. Recruitment limitation, population regulation, and larval connectivity in reef fish metapopulations. Ecology 83, 1104-2093.

Baudisch, J., Bonaventura, L., Iske, A., Miglio, E., 2006. Matrix valued radial basis functions for local vector field reconstruction: applications to computational fluid dynamic models. MOX report n. 75, Tech. rep., MOX, Dipartimento di Matematica F. Brioschi, Politecnico di Milano.

Becker, B.J., Levin, L.A., Fodrie, F.J., McMillan, P.A., 2007. Complex larval connectivity patterns among marine invertebrate populations. Proceedings of the National Academy of Sciences USA 104, 3267-3272.

Berloff, P.S., McWilliams, J.C., 2002. Material transport in oceanic gyres. Part II. Hierarchy of stochastic models. Journal of Physical Oceanography 32, 797-830.

Beverton, R., Holt, S., 1957. On the Dynamics of Exploited Fish Populations. H.M. Stationery Office, London, London.

Biotto, C., 2007. Un modello numerico semi-implicito e semi-lagrangiano per fluidi a superficie libera con forzanti termiche: validazione e applicazioni alla fluidodinamica ambientale. Master Thesis, Politecnico di Milano.

Bode, M., Bode, L., Armsworth, P.R., 2006. Larval dispersal reveals regional sources and sinks in the Great Barrier Reef. Marine Ecology Progress Series 308, 17-35.

Casulli, V., Cattani, E., 1994. Stability, accuracy and efficiency of a semi-implicit method for three-dimensional shallow water flow. Computers and Mathematics with Applications 27 (4), 99-112.

Causin, P., Miglio, E., 2002. Parallel computing for the simulation of 3D free surface flows in environmental applications. Lecture Notes in Computer Science 2474, 78-87.

Chartrand, G., 1985. Introductory Graph Theory. Dover, New York.

Colombini, M., Stocchino, A., 2005. Wind effect in turbulence parametrization Advances in Water Resources 28, 939-949.

Cowen, R.K., Lwiza, K.M.M., Sponaugle, S., Paris, C.B., Olson, B.D., 2000. Connectivity of marine populations: open or closed? Science 287, 857-859.

Cowen, R.K., Paris, C.B., Olson, D.B., Fortuna, J.L., 2003. The role of long distance dispersal versus local retention in replenishing marine populations. Gulf and Caribbean Research 14, 129-137.

Cowen, R.K., Paris, C.B., Srinivasan, A., 2006. Scaling of connectivity in marine populations. Science 311, 522-527.

Cowen, R.K., Sponaugle, S., 2009. Larval dispersal and marine population connectivity. Annual Review of Marine Sciences 1, 443-466.

Dewey, R.K., Stegen, G.R., 1995. A 3-dimensional ocean plume model using stochastic dispersion. In: Thibault, P.A., Bergero, D.M., (Eds.), Proceedings of the Third Annual Conference of the CFD Society of Canada, vol. II, pp. 113-118.

Earn, D.J., Levin, S.A., 2006. Global asymptotic coherence in discrete dynamical systems. Proceedings of the National Academy of Sciences USA 103, 3968-3971.

Earn, D.J., Levin, S.A., Rohani, P., 2000. Coherence and conservation. Science 290, 1360-1364. 
Fields, P.A., Graham, J.B., Rosenblatt, R.H., Somero, G.N., 1993. Effects of expected global climate change on marine faunas. Trends in Ecology and Evolution 8, 361-367.

Fröhlich, C., Brusa, R., 1981. Solar radiation and its variation in time. Solar Physics 74 (1), 209-215.

Galindo, H.M., Olson, D.B., Palumbi, S.R., 2006. Seascape genetics: a coupled oceanographic-genetic model predicts population structure of caribbean corals. Current Biology 16, 1622-1626.

Golub, G., Van Loan, C., 1989. Matrix Computations, second ed. The Johns Hopkins University Press, Baltimore.

Guichard, F., Levin, S.A., Hastings, A., Siegel, D., 2004. Toward a dynamic metacommunity approach to marine reserve theory. BioScience 54, 1003-1011.

Hassell, M.P., 1975. Density-dependence in single-species populations. Journal of Animal Ecology 44, 283-295.

Henderson-Sellers, B., 1986. Calculating the surface energy balance for lake and reservoir modelling: a review. Reviews of Geophysics 24 (3), 625-649.

Imberger, J., Patterson, J.C., 1981. A dynamic reservoir simulation model-DYRESM: 5. In: Fischer, H.B. (Ed.), Transport Models for Inland and Coastal Waters. Academic Press, pp. 310-361.

Iqbal, M., 1983. An Introduction to Solar Radiation. Academic Press, Canada.

James, M.K., Mason, L.B., Armsworth, P.R., Bode, L., 2002. The structure of reef fish metapopulations: modelling larval dispersal and retention patterns. Proceedings of the Royal Society of London B 269, 2079-2086.

Kraft, C.E., Sullivan, P.J., Karateyev, A.Y., Burlakova, L.E., Nekola, J.C., Johnson, L.E., Padilla, D.K., 2002. Landscape patterns of an aquatic invader: assessing dispersal extent from spatial distributions. Ecological Applications 12, 749-759.

Levin, L.A., 2006. Recent progress in understanding larval dispersal: new directions and digressions. Integrative and Comparative Biology 46, 282-297.

Levin, S.A., 1992. The problem of pattern and scale in ecology. Ecology 73, 1943-1967.

Martin, J., McCutcheon, S., 1999. Hydrodynamics and Transport for Water Quality Modeling. Lewis Publishers, U.S.

May, R.M., 1974. Biological population with non-overlapping generations: stable points, stable cycles and chaos. Science 186, 645-647.

Miglio, E., Quarteroni, A., Saleri, F., 1999. Finite element approximation of quasi-3D shallow water equations. Computer Methods in Applied Mechanics and Engineering $174,355-369$.

Miller, T.J., 2007. Contribution of individual-based coupled physicalbiological models to understanding recruitment in marine fish populations. Marine Ecology Process Series 347, 127-138.
Paris, C.B., Chérubin, L.M., Cowen, R.K., 2007. Surfing, spinning, or diving from reef to reef: effects on population connectivity. Marine Ecology Process Series 347, 285-300.

Pielou, E.C., 1977. Mathematical Ecology. Wiley.

Planes, S., Jones, G.P., Thorrold, S.R., 2009. Larval dispersal connects fish populations in a network of marine protected areas. Proceedings of the National Academy of Sciences USA 106, 5693-5697.

Ricklefs, R.E., Miller, G.L., 1999. Ecology. W.H. Freeman and Company, New York.

Rodi, W., 1984. Turbulence models and their application in hydraulics-a state of the art review. Tech. Rep., Institut für Hydromechanik, University of Karlsruhe.

Rose, K.A., Murphy, C.A., Diamond, S.L., Fuiman, L., Thomas, P., 2003. Using nested models and laboratory data for predicting population effects of contaminants on fish: a step toward a bottom-up approach for establishing causality in field studies. Human Ecology Risk Assessment 9, 231-257.

Roughgarden, J., Gaines, S., Possingham, H.P., 1988. Recruitment dynamics in complex life cycles. Science 241, 1460-1466.

Roy, K., Jablonski, D., Valentine, J., 2001. Climate change, species range limits and body size in marine bivalves. Ecology Letters 4, 366-370.

Sammarco, P.W., Andrews, J.C., 1988. Localized dispersal and recruitment in Great Barrier Reef corals: the Helix experiment. Science 239, 1422-1424.

Shank, T.M., Halanych, K.M., 2007. Toward a mechanistic understanding of larva dispersal: insights from genomic fingerprinting of the deep-sea hydrothermal vent tubeworm Riftia pachyptila. Marine Ecology 28, 25-35.

Siegel, D.A., Kinlan, B.P., Gaylord, B., Gaines, S.D., 2003. Lagrangian descriptions of marine larval dispersion. Marine Ecology. Progress Series 260, 83-96.

Siegel, D.A., Mitarai, S., Costello, C.J., Gaines, S.D., Kendall, B.E., Warner, R.R., Winters, K.B., 2008. The stochastic nature of larval connectivity among nearshore marine populations. Proceedings of the National Academy of Sciences USA 105 8974-8979.

Steneck, R.S., 2006. Staying connected in a turbulent world. Science 311, 480-481.

Swinbank, W., 1963. Longwave radiation from clear skies. Quarterly Journal Roya Meteorological Society 89, 339-448.

Turchin, P., 1998. Quantitative Analysis of Movement. Sinauer Associates, Sunderland, UK.

Urban, D., Keitt, T., 2001. Landscape connectivity: a graph-theoretic perspective. Ecology 82, 1205-1218.

Werner, F.E., Cowen, R.K., Paris, C.B., 2007. Coupled biological and physical models Oceanography 20, 54-69. 